\title{
Gauge invariant 1PI effective action for superstring field theory
}

\author{
Ashoke Sen \\ Harish-Chandra Research Institute, \\ Chhatnag Road, Jhusi, Allahabad 211019, India \\ E-mail: sen@mri.ernet.in
}

\begin{abstract}
We construct gauge invariant 1PI effective action for the NS sector of type II and heterotic string field theory. By construction, zero eigenvalues of the kinetic operator of this action determine the renormalized physical masses, and tree level amplitudes computed from this action (after gauge fixing) give the loop corrected S-matrix elements. Using this formalism we can give a simple proof of the result that the renormalized physical masses do not depend on the choice of local coordinate system and locations of picture changing operators used in defining the off-shell amplitude. We also eliminate the need for an infrared regulator in dealing with tadpoles of massless fields.
\end{abstract}

KEYwords: Superstrings and Heterotic Strings, String Field Theory

ARXIV EPRINT: 1411.7478 


\section{Contents}

1 Introduction 1

$\begin{array}{llr}2 & \text { Background } & 3\end{array}$

3 The 1PI effective action $\quad 8$

3.1 The \{\} and [ ] products 9

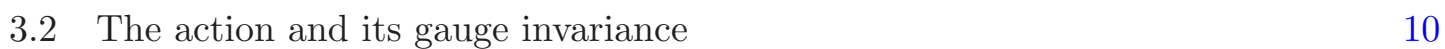

$\begin{array}{ll}3.3 & \text { Equations of motion and the vacuum } \\ & 12\end{array}$

$\begin{array}{ll}3.4 & \text { Effect of changing the local coordinates and/or PCO locations } \\ \end{array}$

4 Applications $\quad 18$

$\begin{array}{ll}\text { 4.1 Mass renormalization } & 19\end{array}$

4.2 Relation to Siegel gauge analysis 22

4.3 S-matrix 25

4.4 Vacuum shift 25

$\begin{array}{ll}\text { A Issues with vertical segment } & 26\end{array}$

\section{Introduction}

Construction of a manifestly Lorentz invariant field theory for heterotic or type II strings has been an open problem. The problem essentially comes from the Ramond (R) sector - there is no natural, fully consistent candidate for the kinetic term of $\mathrm{R}$ sector fields. In contrast in the Neveu-Schwarz (NS) sector one can write down a consistent field theory at the tree level [1-14]. However since in quantum theory $\mathrm{R}$ sector states propagate in the loop even if the external states are all NS sector states, absence of a tree level string field theory including the $\mathrm{R}$ sector states constitutes a bottleneck in the construction of a string field theory at the quantum level.

The purpose of this note is to circumvent this problem by introducing the notion of gauge invariant one particle irreducible (1PI) effective action for string field theory involving NS sector external states. As in conventional field theory, the full quantum amplitudes are given by the tree level amplitudes computed from this 1PI effective action. Thus if we are working with external states in the NS sector, we do not need to worry about the R sector states - they have already been integrated out in constructing this 1PI action.

In a quantum field theory the construction of the 1PI action requires fixing a gauge, computing the 1PI amplitudes using the propagators and vertices of the gauge fixed theory, recasting the result in terms of an 1PI action, and then expressing the result as a sum of a gauge invariant effective action and a gauge fixing term. It turns out that string theory 
allows us to bypass all the steps and directly construct a gauge invariant 1PI action. Of course the final S-matrix elements computed from this are still expressed as integrals over the moduli spaces of Riemann surfaces, and so the construction of this 1PI action does not simplify this computation. However since the 1PI action can be used to ask questions which require going off-shell in the intermediate stage, e.g. the issues of mass renormalization $[15,16]$ and vacuum shift [17] studied recently, using the 1PI effective action we can simplify the analysis of these questions.

Our construction of the 1PI effective action will be based on the definition of the offshell amplitude given in [18] using the formalism of picture changing operators (PCO's) [1922 ]. On-shell there is also a more geometric approach based on integration over supermoduli space [23-41]. Off-shell generalizations of this formalism was attempted in [23-25], but, to our knowledge, a fully satisfactory formalism does not yet exist. If such a formalism is developed then it can also in principle be used to give a different construction of the 1PI effective action.

The 1PI action we have introduced here leads us to the same definition of off-shell amplitudes as the ones used in [15-17]. The main advantage of using the formalism of gauge invariant 1PI action is that using this we can simplify the proof that various physical quantities computed from off-shell amplitudes are independent of the choice of the local coordinates or the locations of the PCO's used in defining the off-shell amplitude. We list below some of the concrete results coming out of this formalism:

1. In the presence of massless fields, conventional string perturbation theory requires an infrared cut-off to regulate contributions from separating type degenerations at the intermediate stages of calculation [33]. Our approach based on gauge invariant 1PI effective action eliminates the need of such an infrared cut-off by subtracting off the contribution from massless fields to the propagator using a well defined procedure.

2. It has been known for a while $[33,42]$ that if we change the local coordinate system and/or PCO locations, then in order to keep the physical quantities (including conventional on-shell S-matrix elements) unchanged we need to make appropriate shifts of various moduli fields. The formalism based on 1PI effective action provides a natural explanation of this result by using the fact that two different 1PI effective action corresponding to different choices of local coordinate systems and/or PCO locations are related by a field redefinition and this field redefinition in general produces a shift in the moduli fields.

3. ref. [16] described a general procedure for computing renormalized masses of physical states using the Siegel gauge kinetic term. However for general states a direct proof of the fact that these renormalized masses are invariant under a change of local coordinate system and/or PCO locations was lacking. We give a proof of this by relating the Siegel gauge analysis to the analysis of the kinetic operator of the gauge invariant 1PI effective action. Since under a change in the local coordinates / PCO locations the kinetic operator of the 1PI action gets conjugated by a non-singular 
matrix, the locations of its zero eigenvalues in the momentum space, representing physical states, remain unchanged.

4. ref. [17] described a general procedure of computing string theory amplitudes around a vacuum that is not the perturbative vacuum but is obtained from it by a small shift of a scalar field. Using the 1PI effective action one can give a justification of these rules. Also unlike in the approach described in [17] where one needs an infrared regulator at the intermediate stages of the calculation for regulating massless tadpoles, in the approach based on 1PI effective action we determine the vacuum expectation values of the massless fields by solving their equations of motion, and hence avoid the appearance of tadpoles even at the intermediate steps of the calculation.

The rest of the paper is organised as follows. In section 2 we review the background material needed for the construction of the 1PI action. In section 3 we describe the 1PI action, its gauge invariance, equations of motions and the construction of the vacuum solutions. We also show that the change in the 1PI action under a change in the choice of local coordinates at the punctures and locations of the PCO's used in the construction of the 1PI action can be absorbed into a redefinition of the string fields. Most of the results in this section are adaptations of the corresponding results in classical bosonic string field theory [43-49] in this new context. In section 4 we apply this formulation to study mass renormalization and vacuum shift effects in string theory. In particular we give a simple argument showing that the renormalized physical mass and the S-matrix elements in perturbative vacuum are independent of the choice of local coordinate system and the PCO locations used to construct the 1PI action. This argument can also be extended to the shifted vacuum when the latter is the correct ground state, Finally in appendix A we extend the analysis of section 3.4 to show that even when we consider two choices of PCO locations which differ in their vertical segment, there is a field redeinition that relates the corresponding 1PI actions.

\section{Background}

In this section we shall review some of the background material that goes into the construction of the 1PI effective action of string field theory. For definiteness we focus on the NS sector of the heterotic string theory but the results have straightforward generalization to the NSNS sector of type II string theories. We shall follow the conventions of [18]. The world sheet theory contains a matter superconformal field theory with central charge $(26,10)$, and a ghost system of total central charge $(-26,-10)$ containing anti-commuting $b, c, \bar{b}, \bar{c}$ ghosts and commuting $\beta, \gamma$ ghosts. The $(\beta, \gamma)$ system can be bosonized as [19]

$$
\gamma=\eta e^{\phi}, \quad \beta=\partial \xi e^{-\phi}, \quad \delta(\gamma)=e^{-\phi}, \quad \delta(\beta)=e^{\phi},
$$

where $\xi, \eta$ are fermions and $\phi$ is a scalar with background charge. We assign (ghost number, picture number, GSO) quantum numbers to various fields as follows:

$$
\begin{aligned}
& c, \bar{c}:(1,0,+), \quad b, \bar{b}:(-1,0,+), \quad \gamma:(1,0,-), \quad \beta:(-1,0,-), \\
& \xi:(-1,1,+), \quad \eta:(1,-1,+), \quad e^{q \phi}:\left(0, q,(-1)^{q}\right) .
\end{aligned}
$$


We do not make any specific choice of the matter superconformal field theory except that it has a free part describing Minkowski space-time so that we have the notion of mass spectrum, S-matrix etc.

We now introduce a vector space $\mathcal{H}_{0}$ containing a subset of states in the matter-ghost conformal field theory satisfying the following conditions:

$$
|s\rangle \in \mathcal{H}_{0} \quad \text { iff } \quad b_{0}^{-}|s\rangle=0, \quad L_{0}^{-}|s\rangle=0, \quad \eta_{0}|s\rangle=0, \quad \text { picture number of }|s\rangle=-1,
$$

where

$$
b_{0}^{ \pm} \equiv\left(b_{0} \pm \bar{b}_{0}\right), \quad L_{0}^{ \pm} \equiv\left(L_{0} \pm \bar{L}_{0}\right) .
$$

The off-shell string field is taken to be a state $|\Psi\rangle \in \mathcal{H}_{0}$ of ghost number 2. For later use we also define

$$
c_{0}^{ \pm}=\frac{1}{2}\left(c_{0} \pm \bar{c}_{0}\right) .
$$

Finally we denote by $Q_{B}$ the BRST charge and by $\mathcal{X}(z)=\left\{Q_{B}, \xi(z)\right\}$ the picture changing operator.

Since our construction will make use of some of the results of [18], we review here the pertinent results. We denote by $\mathcal{M}_{g, n}$ the moduli space of Riemann surfaces of genus $g$ with $n$ distinguishable punctures. $\widetilde{\mathcal{P}}_{g, n}$ will denote a fiber bundle over $\mathcal{M}_{g, n}$ where the fiber is infinite dimensional, containing information about a choice of local coordinate system (up to phases) around each puncture and locations of $2 g+n-2$ PCO's on the Riemann surface. ${ }^{1}$ In [18] we constructed, for a set of states $\left\{\left|\Phi_{1}\right\rangle, \cdots\left|\Phi_{n}\right\rangle\right\} \in \mathcal{H}_{0}$, a $p$-form $\Omega_{p}^{(g, n)}\left(\left|\Phi_{1}\right\rangle, \cdots\left|\Phi_{n}\right\rangle\right)$ on $\widetilde{\mathcal{P}}_{g, n}$ for arbitrary $p$, satisfying

$$
\begin{aligned}
& \sum_{i=1}^{n}(-1)^{n_{1}+\cdots n_{i-1}} \Omega_{p}^{(g, n)}\left(\left|\Phi_{1}\right\rangle, \cdots\left|\Phi_{i-1}\right\rangle, Q_{B}\left|\Phi_{i}\right\rangle,\left|\Phi_{i+1}\right\rangle, \cdots\left|\Phi_{n}\right\rangle\right)= \\
&(-1)^{p} d \Omega_{p-1}^{(g, n)}\left(\left|\Phi_{1}\right\rangle, \cdots,\left|\Phi_{n}\right\rangle\right),
\end{aligned}
$$

where $d$ denotes exterior derivative on $\widetilde{\mathcal{P}}_{g, n}$ and $n_{i}$ is the ghost number of $\left|\Phi_{i}\right\rangle$. $\Omega_{p}^{(g, n)}\left(\left|\Phi_{1}\right\rangle, \cdots\left|\Phi_{n}\right\rangle\right)$ is a multilinear function of the $n$ states $\left|\Phi_{1}\right\rangle, \cdots\left|\Phi_{n}\right\rangle$, constructed in terms of a correlation function on the Riemann surface with the state $\left|\Phi_{i}\right\rangle$ inserted at the $i$-th puncture using the local coordinate system at that puncture. It has the symmetry property

$s_{i, i+1} \circ \Omega_{p}^{(g, n)}\left(\left|\Phi_{1}\right\rangle, \cdots\left|\Phi_{i-1}\right\rangle,\left|\Phi_{i+1}\right\rangle,\left|\Phi_{i}\right\rangle,\left|\Phi_{i+2}\right\rangle \cdots\left|\Phi_{n}\right\rangle\right)=(-1)^{n_{i} n_{i+1}} \Omega_{p}^{(g, n)}\left(\left|\Phi_{1}\right\rangle, \cdots\left|\Phi_{n}\right\rangle\right)$

where $s_{i, i+1}$ is the transformation on $\widetilde{\mathcal{P}}_{g, n}$ that exchanges the punctures $i$ and $i+1$ together with their local coordinates and $s_{i, i+1} \circ \Omega_{p}^{(g, n)}$ is the pullback of $\Omega_{p}^{(g, n)}$ under this transformation. Another useful property of $\Omega_{p}^{(g, n)}\left(\left|\Phi_{1}\right\rangle, \cdots\left|\Phi_{n}\right\rangle\right)$ is that it is non-vanishing only if the ghost numbers of $\left|\Phi_{1}\right\rangle, \cdots\left|\Phi_{n}\right\rangle$ add up to $p-6 g+6$.

\footnotetext{
${ }^{1}$ This is a generalization of corresponding construction in bosonic string theory $[47,50]$ where the choice of local coordinate system is the only data on the fiber.
} 
The analysis of $[15,16,18]$ also identified, for each $g, n$, a $6 g-6+2 n$ dimensional subspace $\mathcal{K}_{g, n}$ of $\mathcal{M}_{g, n}$ called the 1 PI region and a 'section' $\mathcal{R}_{g, n}$ of $\widetilde{\mathcal{P}}_{g, n}$ over this subspace with the following properties ${ }^{2}$

1. For some $(g, n), \mathcal{R}_{g, n}$ could have 'vertical segments' along which the locations of the PCO's change without any change in the moduli parametrizing the base. This is necessary for avoiding the spurious singularities which occur in type II / heterotic string perturbation theory [20]. The rules for carrying out the integration along these vertical segments have been described in [18] and further refined in [51]. For this reason we shall refer to $\mathcal{R}_{g, n}$ as an integration cycle instead of a section.

2. Even though the punctures are distinguishable, we choose $\mathcal{R}_{g, n}$ to be symmetric under the exchange of the punctures. This means that for every point in $\mathcal{R}_{g, n}$ we'll also have in $\mathcal{R}_{g, n}$ the point obtained by exchanging any two punctures together with their local coordinate systems, and the locations of the PCO's will remain unchanged under this exchange.

3. Some time we may encounter a situation in which a single integration cycle cannot be chosen consistent with this symmetry principle. A simple example is three punctured sphere requiring one insertion of the picture changing operator. In order to be consistent with the symmetry principle we would require that the location of the $\mathrm{PCO}$ will be invariant under any $\mathrm{SL}(2, C)$ transformation that permutes these three punctures. It is easy to verify that there is no such point on the sphere. However we can choose a pair of points which are permuted among themselves under the $\operatorname{SL}(2, C)$ transformations that permute the three punctures. Thus we can restore full permutation symmetry by taking the average of these two choices for the location of the PCO. To deal with such situations we allow $\mathcal{R}_{g, n}$ to be formal weighted average of multiple integration cycles. Since eventually we shall be interested in integrating $\Omega_{6 g-6+2 n}^{(g, n)}$ over these integration cycles, the integral over the weighted average of integration cycles can be regarded as the weighed average of the integrals over the corresponding integration cycles.

4. Take a Riemann surface (equipped with choice of local coordinate system and arrangement of PCO's) corresponding to a point in $\mathcal{R}_{g_{1}, n_{1}}$ and another Riemann surface corresponding to a point in $\mathcal{R}_{g_{2}, n_{2}}$. If we take one puncture from each of these Riemann surfaces, denote the local coordinates around these punctures by $z$ and $w$ with the punctures being located at $z=0$ and $w=0$, and glue the two Riemann

\footnotetext{
${ }^{2}$ In $[15,16,18]$ we did not use the specific symbols $\mathcal{K}_{g, n}$ and $\mathcal{R}_{g, n}$ for these subspaces. This notation is introduced in this paper. Also, while the concrete algorithm for constructing these regions was given in $[15,16,18]$, explicit construction of these regions was not given as there is a lot of freedom. A concrete example can be provided by computing the off-shell 1PI amplitudes in the closed bosonic string field theory of [47] in Siegel gauge. The subspace of the moduli space covered by the 1PI amplitudes will give $\mathcal{K}_{g, n}$ and the local coordinates at the punctures induced from this construction will give $\mathcal{R}_{g, n}$ sans the information on the PCO's. The PCO locations will then have to be chosen consistent with gluing compatibility and avoiding spurious singularities using the trick of vertical integration described in [18].
} 
surfaces by the plumbing fixture relation

$$
z w=e^{-s+i \theta}, \quad 0 \leq s<\infty, \quad 0 \leq \theta<2 \pi,
$$

we get a two parameter family of Riemann surfaces of genus $g_{1}+g_{2}$ and $n_{1}+n_{2}-2$ punctures, labelled by $s$ and $\theta$. Considering the $6 g_{i}-6+2 n_{i}$ parameter family of Riemann surfaces contained in $\mathcal{R}_{g_{i}, n_{i}}$ we get altogether $6\left(g_{1}+g_{2}\right)+2\left(n_{1}+n_{2}\right)-10$ parameter family of Riemann surfaces. These describe a subspace of $\mathcal{M}_{g_{1}+g_{2}, n_{1}+n_{2}-2}$ which we shall denote by $\mathcal{K}_{g_{1}, n_{1}} \circ \mathcal{K}_{g_{2}, n_{2}}$. Furthermore the choice of local coordinates at the punctures and the locations of PCO's on the original surfaces automatically produce similar data on the final Riemann surfaces. Thus we really have a $6\left(g_{1}+\right.$ $\left.g_{2}\right)+2\left(n_{1}+n_{2}\right)-10$ dimensional subspace of $\widetilde{\mathcal{P}}_{g_{1}+g_{2}, n_{1}+n_{2}-2}$. We shall call this $\mathcal{R}_{g_{1}, n_{1}} \circ \mathcal{R}_{g_{2}, n_{2}}$. Summing over all inequivalent permutations of the external punctures we generate a subspace of $\widetilde{\mathcal{P}}_{g_{1}+g_{2}, n_{1}+n_{2}-2}$ that we shall call $\mathbf{S}\left[\mathcal{R}_{g_{1}, n_{1}} \circ \mathcal{R}_{g_{2}, n_{2}}\right]$.

5. Similarly by gluing three families of Riemann surfaces associated with $\mathcal{R}_{g_{1}, n_{1}}, \mathcal{R}_{g_{2}, n_{2}}$ and $\mathcal{R}_{g_{3}, n_{3}}$ in all possible ways, but using only two plumbing fixtures so that no closed loop is formed, we generate a new $6\left(g_{1}+g_{2}+g_{3}\right)+2\left(n_{1}+n_{2}+n_{3}\right)-14$ parameter family of Riemann surfaces of genus $g_{1}+g_{2}+g_{3}$ and $n_{1}+n_{2}+n_{3}-4$ punctures. These correspond to a subspace of $\mathcal{M}_{g_{1}+g_{2}+g_{3}, n_{1}+n_{2}+n_{3}-4}$. Again the choice of the local coordinates and the PCO data on the original Riemann surfaces generate similar data on the final Riemann surfaces, producing a subspace of $\widetilde{\mathcal{P}}_{g_{1}+g_{2}+g_{3}, n_{1}+n_{2}+n_{3}-4}$. This process can be continued, generating more and more families of Riemann surfaces by gluing more and more 1PI families of Riemann surfaces in all possible inequivalent ways.

6. Now consider a given $g$ and $n$. Following the procedure described above we can generate a large class of Riemann surfaces of genus $g$ with $n$ punctures, - some from 1 PI region $\mathcal{K}_{g, n}$ of the moduli space, some from gluing two 1PI family of Riemann surfaces of lower genera and/or lower number of punctures and so on. The claim is that this exhausts the whole of $\mathcal{M}_{g, n}$ in a one to one fashion, - indeed $\mathcal{K}_{g, n}$ is chosen so as to precisely account for any deficit that we might have from gluing surfaces of lower genera / lower number of punctures. Furthermore the gluing compatible choice of integration cycles guarantee that the choice of the integration cycle over $\mathcal{M}_{g, n}$ that we get this way is continuous across the boundaries between different types of contributions e.g. the boundary of $\mathcal{R}_{g_{1}, n_{1}} \circ \mathcal{R}_{g-g_{1}, n-n_{1}+2}$ from the $s=0$ end of the plumbing fixture relation (2.8) smoothly matches a component of the boundary of $\mathcal{R}_{g, n}$. Again, the construction of $\mathcal{R}_{g, n}$ is designed to ensure this by choosing the integration cycle $\mathcal{R}_{g, n}$ on $\mathcal{K}_{g, n}$ such that it smoothly matches the integration cycle $\mathcal{R}_{g_{1}, n_{1}} \circ \mathcal{R}_{g-g_{1}, n-n_{1}+2}$ on $\mathcal{K}_{g_{1}, n_{1}} \circ \mathcal{K}_{g-g_{1}, n-n_{1}+2}$ at the common boundary of $\mathcal{K}_{g, n}$ and $\mathcal{K}_{g_{1}, n_{1}} \circ \mathcal{K}_{g-g_{1}, n-n_{1}+2}$. We shall denote the integration cycle of $\widetilde{\mathcal{P}}_{g, n}$ over the whole of $\mathcal{M}_{g, n}$ obtained this way by $\mathcal{S}_{g, n}$. Also all parts of $\mathcal{S}_{g, n}$ outside $\mathcal{R}_{g, n}$ will be called one particle reducible (1PR) part of $\mathcal{S}_{g, n}$ and the associated Riemann surfaces will be called 1PR Riemann surfaces. 
7. In this description of $\mathcal{M}_{g, n}$, all separating type degenerations - where a Riemann surface degenerates into two or more distinct Riemann surfaces joined by a long handle - comes from 1PR Riemann surfaces with the parameter $s$ in (2.8) approaching infinity for one or more of the plumbing fixture. Thus the 1 PI family $\mathcal{R}_{g, n}$ does not contain any boundary associated with separating type degeneration. However $\mathcal{R}_{g, n}$ can (and does) contain boundaries corresponding to non-separating type degenerations where two punctures on the same Riemann surface are connected by a long handle.

Once these different regions have been identified, we define the genus $g, n$-point off-shell amplitude with external states $\left|\Phi_{1}\right\rangle, \cdots\left|\Phi_{n}\right\rangle$ to be the integral of $\Omega_{6 g-6+2 n}^{(g, n)}\left(\left|\Phi_{1}\right\rangle, \cdots\left|\Phi_{n}\right\rangle\right)$ over the subspace $\mathcal{S}_{g, n}$. The contribution to this integral from the 1PI part $\mathcal{R}_{g, n}$ of $\mathcal{S}_{g, n}$ will be called the 1PI contribution to the off-shell amplitude, while the contribution from the $1 \mathrm{PR}$ part of $\mathcal{S}_{g, n}$ will be called the $1 \mathrm{PR}$ contribution. This will be justified later when we construct the 1PI effective action for the string field theory and compute the off-shell amplitude from this effective action. We should note here that off-shell amplitudes refer to off-shell Green's functions with external tree level propagators truncated.

Readers familiar with the construction of the covariant string field theory [47] would recognize the close resemblance between the regions $\mathcal{R}_{g, n}$ used here and the regions $\mathcal{V}_{g, n}$ used in the construction of the quantum master action of bosonic string field theory. Of course one difference is that in the construction of [47] there was no information about the PCO's, so the regions $\mathcal{V}_{g, n}$ were subspaces of $\widehat{\mathcal{P}}_{g, n}$ which had $\mathcal{M}_{g, n}$ as the base and the data on local coordinates (modulo phases) at the punctures as fibers. The main difference however is that while building the analog of the integration cycle $\mathcal{S}_{g, n}$ over the whole of $\mathcal{M}_{g, n}$ from the plumbing fixture of the Riemann surfaces associated with $\mathcal{V}_{g, n}$, we also allow closed loops. Thus for example we can glue two punctures on the same Riemann surface associated with $\mathcal{V}_{g, n}$, glue two punctures of a Riemann surface associated with $\mathcal{V}_{g_{1}, n_{1}}$ with two punctures of a Riemann surface associated with $\mathcal{V}_{g_{2}, n_{2}}$ and so on. As a result all degenerations - separating as well as non-separating type - come from one or more of the plumbing fixture parameters $s$ approaching infinity, and $\mathcal{V}_{g, n}$ never contains any degenerate Riemann surface. If we take the $\mathcal{V}_{g, n}$ 's of [47] and glue them together in all possible ways using (2.8) including configuration with loops, but not allowing those configurations where by taking the parameter $s$ associated with one of the plumbing fixture relations to infinity we reach a separating type degeneration, we get possible candidates for $\mathcal{R}_{g, n}$ (without the PCO data). This is precisely analogous to the construction of 1PI amplitudes using Feynman diagrams of a quantum field theory.

The boundary of $\mathcal{R}_{g, n}$ is an object of special interest since the integral of a total derivative on $\mathcal{R}_{g, n}$ will receive contribution from this boundary. As already discussed, $\mathcal{R}_{g, n}$ has boundaries corresponding to non-separating type degenerations. However they will not be of interest to us since the boundary contributions from these boundaries can be made to vanish using appropriate $i \epsilon$ prescription $[52,53]$. Thus from now on we shall ignore the existence of these boundaries. The other boundaries of $\mathcal{R}_{g, n}$ coincide with the boundaries of $\mathcal{R}_{g_{1}, n_{1}} \circ \mathcal{R}_{g-g_{1}, n-n_{1}+2}$ arising from the $s=0$ end of the plumbing fixture relation (2.8) 
since $\mathcal{R}_{g, n}$ and $\mathcal{R}_{g_{1}, n_{1}} \circ \mathcal{R}_{g-g_{1}, n-n_{1}+2}$ will have to 'fit together' inside $\widetilde{\mathcal{P}}_{g, n}$ to generate part of the smooth integration cycle $\mathcal{S}_{g, n}$. Denoting this boundary by $\left\{\mathcal{R}_{g_{1}, n_{1}}, \mathcal{R}_{g_{2}, n_{2}}\right\}$ we get

$$
\partial \mathcal{R}_{g, n}=-\frac{1}{2} \sum_{\substack{g_{1}, g_{2}=\\ g_{1}+g_{2}=g}} \sum_{\substack{n_{1}, n_{2} \\ n_{1}+n_{2}=n+2}} \mathbf{S}\left[\left\{\mathcal{R}_{g_{1}, n_{1}}, \mathcal{R}_{g_{2}, n_{2}}\right\}\right],
$$

where, as before, $\mathbf{S}$ denotes the operation of summing over all inequivalent permutation of the external punctures. The factor of $1 / 2$ reflects that on the right hand the same term appears twice due to the exchange symmetry $g_{1} \leftrightarrow g_{2}, n_{1} \leftrightarrow n_{2}$. Even for $g_{1}=g_{2}$ and $n_{1}=n_{2}$ there is a double counting since $\mathbf{S}$ sums over exchanges of the puncture labels. The - sign reflects that the boundaries of $\mathcal{R}_{g, n}$ and $\mathcal{R}_{g_{1}, n_{1}} \circ \mathcal{R}_{g_{2}, n_{2}}$ must be oppositely oriented since $\mathcal{R}_{g, n}$ together with $(1 / 2) \sum_{g_{1}+g_{2}=g} \sum_{n_{1}+n_{2}=n+2}^{n_{1}, n_{2}} \mathcal{R}_{g_{1}, n_{1}} \circ \mathcal{R}_{g_{2}, n_{2}}$ fill part of $\mathcal{S}_{g, n}$. Physically $\left\{\mathcal{R}_{g_{1}, n_{1}}, \mathcal{R}_{g_{2}, n_{2}}\right\}$ represents the set of punctured Riemann surfaces equipped with choice of local coordinates at the punctures and PCO locations that we obtain by gluing the families of Riemann surfaces corresponding to $\mathcal{R}_{g_{1} . n_{1}}$ and $\mathcal{R}_{g_{2}, n_{2}}$ using plumbing fixture relation (2.8) with the parameter $s$ set to zero. The orientation of $\{A, B\}$ will be defined by taking its volume form to be $d \theta \wedge d V_{A} \wedge d V_{B}$ where $d V_{A}$ and $d V_{B}$ are volume forms on $A$ and $B$ respectively.

It follows from general properties of conformal field theories on Riemann surfaces that on $\left\{\mathcal{R}_{g_{1}, n_{1}}, \mathcal{R}_{g_{2}, n_{2}}\right\}, \Omega_{p}^{\left(g_{1}+g_{2}, n_{1}+n_{2}-2\right)}$ satisfies the factorization property

$$
\begin{aligned}
& \int_{\theta} \Omega_{p}^{\left(g_{1}+g_{2}, n_{1}+n_{2}-2\right)}\left(\left|\Phi_{1}\right\rangle, \cdots\left|\Phi_{n_{1}+n_{2}-2}\right\rangle\right) \\
&=\sum_{\substack{p_{1}, p_{2} \\
p_{1}+p_{2}=p-1}}(-1)^{n_{1}+\cdots n_{n_{1}-1}+p_{1}+p_{2}+p_{1} p_{2}} \Omega_{p_{1}}^{\left(g_{1}, n_{1}\right)}\left(\left|\Phi_{1}\right\rangle, \cdots\left|\Phi_{n_{1}-1}\right\rangle,\left|\varphi_{r}\right\rangle\right) \\
& \qquad \Omega_{p_{2}}^{\left(g_{2}, n_{2}\right)}\left(\left|\varphi^{r}\right\rangle,\left|\Phi_{n_{1}}\right\rangle, \cdots\left|\Phi_{n_{1}+n_{2}-2}\right\rangle\right)
\end{aligned}
$$

where $\int_{\theta}$ denotes the result of integration over the angular coordinate $\theta$ appearing in the plumbing fixture relation (2.8), and $\left\{\left|\varphi_{r}\right\rangle\right\}$ and $\left\{\left|\varphi^{r}\right\rangle\right\}$ are a set of dual basis of $\mathcal{H}_{0}$ satisfying

$$
\left\langle\varphi^{r}\left|c_{0}^{-}\right| \varphi_{s}\right\rangle=\delta^{r}{ }_{s} \quad \Leftrightarrow \quad\left\langle\varphi_{s}\left|c_{0}^{-}\right| \varphi^{r}\right\rangle=\delta^{r}{ }_{s} .
$$

In arriving at (2.10) one has to use the fact that the $\theta$ integral produces the projector $\delta_{L_{0}, \bar{L}_{0}}$ and is accompanied by an insertion of $b_{0}^{-}$in the correlator. Together they ensure that the sum over the complete set of states $\left|\varphi_{r}\right\rangle,\left|\varphi^{r}\right\rangle$ run only over states in $\mathcal{H}_{0}$ satisfying (2.3). The $(-1)^{n_{1}+\cdots n_{n_{1}-1}+p_{1}+p_{2}+p_{1} p_{2}}$ factor can be obtained from the results of [18] after taking into account an extra minus sign due to the fact that here we are picking the boundary contribution from the lower limit of the $s$ integral while [18] analyzed the boundary contribution from the upper limit. We also need to use the fact that the basis states $\left\langle\varphi_{r}^{c}\right|$ of [18] are related to $\left\langle\varphi^{r}\right|$ given here by $\left\langle\varphi_{r}^{c}\right|=\left\langle\varphi^{r}\right| c_{0}^{-}$.

\section{The 1PI effective action}

The construction of the 1PI action of NS sector fields will be a generalization of the tree level action for NS sector fields given in [2]. Most of the properties of this theory discussed 
in this section will involve adapting the various results for the classical bosonic string field theory of [44-46] to the 1PI effective action of heterotic / type II string field theory. However we shall try to keep the discussion self-contained by briefly outlining the proofs of the various results we shall describe.

We shall take the string field to be an element of the small Hilbert space as given by the $\eta_{0}|s\rangle=0$ condition in (2.3)[19]. This was the spirit of the construction in [1,2], and some of the recent approaches, e.g. the ones considered in $[11,12,14]$ also falls broadly in this class. There are also alternate approaches based on taking the string field to be an element of the large Hilbert space, e.g. the ones used in [3-7] — but at present we do not know whether this approach can be generalized to give a fully consistent 1PI effective action for the NS sector fields.

\subsection{The \{\} and [ ] products}

We define, for $\left|\Phi_{i}\right\rangle \in \mathcal{H}_{0}$,

$$
\left\{\Phi_{1} \cdots \Phi_{n}\right\}=\sum_{g=0}^{\infty}\left(g_{s}\right)^{2 g} \int_{\mathcal{R}_{g, n}} \Omega_{6 g-6+2 n}^{(g, n)}\left(\left|\Phi_{1}\right\rangle, \cdots\left|\Phi_{n}\right\rangle\right) .
$$

If $\mathcal{R}_{g, n}$ contains vertical segments then one has to use the procedure described in $[18,51]$ for carrying out the integral over these segments. We also define $\left|\left[\Phi_{2} \cdots \Phi_{n}\right]\right\rangle \in \mathcal{H}_{0}$ via the relations

$$
\left\langle\Phi_{1}\left|c_{0}^{-}\right|\left[\Phi_{2} \cdots \Phi_{n}\right]\right\rangle=\left\{\Phi_{1} \cdots \Phi_{n}\right\}
$$

for all $\left|\Phi_{1}\right\rangle \in \mathcal{H}_{0}$. Here $\langle A \mid B\rangle$ denotes the BPZ inner product. Note that to keep the notation simple we have dropped the ket symbol |\rangle from the states when they appear in the argument of \{\} or [ ]. Also we shall drop the ket symbol from $\left|\left[\Phi_{2} \cdots \Phi_{n}\right]\right\rangle$ except in inner products, i.e. $\left[\Phi_{2} \cdots \Phi_{n}\right]$ will actually denote $\left|\left[\Phi_{2} \cdots \Phi_{n}\right]\right\rangle$. It follows from the property of $\Omega_{p}^{(g, n)}$ that in order to get non-vanishing result for $\left\{\Phi_{1} \cdots \Phi_{n}\right\}$ we must have $\sum_{i=1}^{n} n_{i}=2 n$ where $n_{i}$ is the ghost number of $\Phi_{i}$, and that $\left[\Phi_{2} \cdots \Phi_{n}\right]$ has ghost number equal to $3+\sum_{i=2}^{n} n_{i}-2(n-1)$.

Based on the identities (2.6) and (2.9) one can now establish a set of identities involving $Q_{B}$ and [ ]. Since their derivation is identical to the ones for bosonic string field theory [47] we shall be brief. The first identity gives the symmetry properties of $[\cdots]$ and $\{\cdots\}$ :

$$
\begin{aligned}
{\left[\Phi_{2} \cdots \Phi_{i-1} \Phi_{i+1} \Phi_{i} \Phi_{i+2} \cdots \Phi_{n}\right] } & =(-1)^{n_{i} n_{i+1}}\left[\Phi_{2} \cdots \Phi_{n}\right] \\
\left\{\Phi_{1} \Phi_{2} \cdots \Phi_{i-1} \Phi_{i+1} \Phi_{i} \Phi_{i+2} \cdots \Phi_{n}\right\} & =(-1)^{n_{i} n_{i+1}}\left\{\Phi_{1} \Phi_{2} \cdots \Phi_{n}\right\} .
\end{aligned}
$$

The inner product of the first equation of (3.3) with an arbitrary bra $\left\langle\Phi_{1}\right| c_{0}^{-}$, which is also the second equation of (3.3), comes from the integral of (2.7) over $\mathcal{R}_{g, n}$. The second useful identity is

$$
\left\{\Phi_{1} \cdots \Phi_{k}\left[\widetilde{\Phi}_{1} \cdots \widetilde{\Phi}_{\ell}\right]\right\}=-\left\{\widetilde{\Phi}_{1} \cdots \widetilde{\Phi}_{\ell}\left[\Phi_{1} \cdots \Phi_{k}\right]\right\} \quad \text { for }\left|\Phi_{i}\right\rangle,\left|\widetilde{\Phi}_{j}\right\rangle \in \mathcal{H}_{0} .
$$

To prove this we note that as a consequence of (2.11), $\sum_{r}\left|\varphi_{r}\right\rangle\left\langle\varphi^{r}\right| c_{0}^{-}$and $\sum_{r}\left|\varphi^{r}\right\rangle\left\langle\varphi_{r}\right| c_{0}^{-}$ act as identity operator on states in $\mathcal{H}_{0}$. If we insert the first operator in front of $[\cdots]$ on 
the left hand side of (3.4) and the second operator in front of $[\cdots]$ on the right hand side of (3.4), we see that the two expressions are identical except for a sign and the locations of $\varphi_{r}$ and $\varphi^{r}$ in the correlation function. Using the ghost number conservation laws mentioned below (3.2), the fact that ghost numbers of $\varphi_{r}$ and $\varphi^{r}$ are related by $n_{\varphi_{r}}=5-n_{\varphi^{r}}$, and (3.3) we can show that the arguments inside $\{\cdots\}$ on the two sides can be brought to the same arrangement at the cost of generating an extra minus sign. This cancels the explicit minus sign in (3.4) showing that the two sides are equal.

The third equation, known as the main identity [47], tells us that for $n \geq 1,{ }^{3}$

$$
\begin{aligned}
& Q_{B}\left[\Phi_{2} \cdots \Phi_{n}\right]+\sum_{i=2}^{n}(-1)^{n_{2}+\cdots n_{i-1}}\left[\Phi_{2} \cdots \Phi_{i-1} Q_{B} \Phi_{i} \Phi_{i+1} \cdots \Phi_{n}\right] \\
& +\sum_{\substack{\ell, k \geq 0 \\
\ell+k=n-1}} \sum_{\substack{\left\{i_{r} ; r=1, \ldots \ell\right\},\left\{j_{s} ; s=1, \cdots k\right\} \\
\left\{i_{r}\right\} \cup\left\{j_{s}\right\}=\{2, \cdots n\}}} \sigma\left(\left\{i_{r}\right\},\left\{j_{s}\right\}\right)\left[\Phi_{i_{1}} \cdots \Phi_{i_{\ell}}\left[\Phi_{j_{1}} \cdots \Phi_{j_{k}}\right]\right]=0
\end{aligned}
$$

where in the last term the sum runs over all possible ways of splitting the set $\{2, \cdots n\}$ into the set $\left\{i_{r}\right\}$ and the set $\left\{j_{s}\right\} . \sigma\left(\left\{i_{r}\right\},\left\{j_{s}\right\}\right)$ is the sign that one picks up while rearranging $Q_{B}, \Phi_{2}, \cdots \Phi_{n}$ to $\Phi_{i_{1}}, \cdots \Phi_{i_{\ell}}, Q_{B}, \Phi_{j_{1}}, \cdots \Phi_{j_{k}}$. The inner product of (3.5) with an arbitrary bra $\left\langle\Phi_{1}\right| c_{0}^{-}$is obtained by integrating (2.6) over $\mathcal{R}_{g, n}$ and multiplying both sides by $(-1)^{n_{1}}$. The first two terms in (3.5) come from the integral of the left hand side of (2.6) whereas the last term has its origin in the integral of the right hand side of (2.6). The latter, being a total derivative, receives contribution from the boundary $\partial \mathcal{R}_{g, n}$ described in (2.9). This can then be evaluated using (2.10). The terms that are generated are of two types: $\left\{\Phi_{1} \Phi_{i_{1}} \cdots \Phi_{i_{\ell}}\left[\Phi_{j_{1}} \cdots \Phi_{j_{k}}\right]\right\}$ and $\left\{\Phi_{j_{1}} \cdots \Phi_{j_{k}}\left[\Phi_{1} \Phi_{i_{1}} \cdots \Phi_{i_{\ell}}\right]\right\}$. Using (3.4) we can show that the two terms are in fact identical after performing the sum over the sets $\left\{i_{r}\right\}$ and $\left\{j_{s}\right\}$. This allows us to keep only the first term and cancel the extra factor of $1 / 2$ in (2.10). This can then be interpreted as the inner product of $-\left\langle\Phi_{1}\right| c_{0}^{-}$with the last term on the left hand side of (3.5).

Note that inside $[\cdots]$ in the first term of (3.5) the first argument is $\Phi_{2}$ and hence there are only $n-1$ arguments. Thus for $n=1$ we have the equation $Q_{B}[]+[[]]=0$.

\subsection{The action and its gauge invariance}

We are now ready to describe the 1PI effective action and its gauge invariance. The string field $|\Psi\rangle$ is taken to be an element of $\mathcal{H}_{0}$ of ghost number 2 . The action is given by

$$
S(|\Psi\rangle)=g_{s}{ }^{-2}\left[\frac{1}{2}\left\langle\Psi\left|c_{0}^{-} Q_{B}\right| \Psi\right\rangle+\sum_{n=1}^{\infty} \frac{1}{n !}\left\{\Psi^{n}\right\}\right]
$$

where $\left\{\Psi^{n}\right\}$ denotes $\{\Psi \Psi \cdots \Psi\}$ with $n$ copies of $\Psi$ inside \{\} . The gauge transformation law for $|\Psi\rangle$ is given by

$$
\delta|\Psi\rangle=Q_{B}|\Lambda\rangle+\sum_{n=0}^{\infty} \frac{1}{n !}\left[\Psi^{n} \Lambda\right]
$$

\footnotetext{
${ }^{3}$ In the analysis of [47] there is an additional contribution involving $\Delta$ operation in (2.9) which gives rise to additional terms in the identities involving $Q_{B}$ and [ ]. These terms are absent here since we build up the moduli space $\mathcal{M}_{g, n}$ from 1PI Riemann surfaces instead of the Riemann surfaces associated with the elementary vertices of string field theory.
} 
where $|\Lambda\rangle$ is an element of $\mathcal{H}_{0}$ with ghost number 1. Proof of gauge invariance of the action (3.6) proceeds in the same way as in [44-47] except that in classical string field theory the range of summation over $n$ in (3.6) and (3.7) begins at 3 and 1 respectively. We shall now briefly discuss the proof. Under the transformation (3.7) the change $\delta S$ in the action is given by

$g_{s}^{2} \delta S=\sum_{n=0}^{\infty} \frac{1}{n !}\left\langle\Psi \mid c_{0}^{-} Q_{B}\left[\Psi^{n} \Lambda\right]\right\rangle+\sum_{n=1}^{\infty} \frac{1}{(n-1) !}\left\{\Psi^{n-1} Q_{B} \Lambda\right\}+\sum_{n=1}^{\infty} \frac{1}{(n-1) !} \sum_{m=0}^{\infty} \frac{1}{m !}\left\{\Psi^{n-1}\left[\Psi^{m} \Lambda\right]\right\}$,

where we have used $Q_{B}{ }^{2}=0$ to set one of the terms to 0 . Now the first two terms on the right hand side can be manipulated as

$$
\begin{aligned}
& \sum_{n=0}^{\infty} \frac{1}{n !}\left\langle Q_{B} \Psi \mid c_{0}^{-}\left[\Psi^{n} \Lambda\right]\right\rangle+\sum_{n=1}^{\infty} \frac{1}{(n-1) !}\left\langle Q_{B} \Lambda\left|c_{0}^{-}\right|\left[\Psi^{n-1}\right]\right\rangle \\
& =\sum_{n=0}^{\infty} \frac{1}{n !}\left\{Q_{B} \Psi \Psi^{n} \Lambda\right\}-\sum_{n=1}^{\infty} \frac{1}{(n-1) !}\left\langle\Lambda \mid c_{0}^{-} Q_{B}\left[\Psi^{n-1}\right]\right\rangle \\
& =-\sum_{n=0}^{\infty} \frac{1}{n !}\left\langle\Lambda\left|c_{0}^{-}\right|\left[Q_{B} \Psi \Psi^{n}\right]\right\rangle-\sum_{n=1}^{\infty} \frac{1}{(n-1) !}\left\langle\Lambda \mid c_{0}^{-} Q_{B}\left[\Psi^{n-1}\right]\right\rangle
\end{aligned}
$$

We now interpret the first term on the right hand side as the average of $n+1$ terms where $Q_{B}$ acts on each of the $\Psi$ 's inside $[\cdots]$ once, make change of variables $n=m-1$ in the first term and $n=m+1$ in the second term and use (3.5) with all the $\Phi_{i}$ 's equal to $\Psi$ to express the right hand side of (3.9) as

$$
\sum_{m=0}^{\infty} \frac{1}{m !} \sum_{\substack{0 \leq n_{1}, n_{2} \leq \infty \\ n_{1}+n_{2}=m}} \frac{m !}{n_{1} ! n_{2} !}\left\langle\Lambda\left|c_{0}^{-}\right|\left[\Psi^{n_{1}}\left[\Psi^{n_{2}}\right]\right]\right.
$$

The $m ! /\left(n_{1} ! n_{2} !\right)$ term comes from the number of ways we can divide the $m$ objects into $n_{1}$ objects and $n_{2}=m-n_{1}$ objects. Regarding $n_{1}, n_{2}$ as independent summation variables we can now express this as

$$
\sum_{n_{1}, n_{2}=0}^{\infty} \frac{1}{n_{1} ! n_{2} !}\left\{\Lambda \Psi^{n_{1}}\left[\Psi^{n_{2}}\right]\right\}=-\sum_{n_{1}, n_{2}=0}^{\infty} \frac{1}{n_{1} ! n_{2} !}\left\{\Psi^{n_{2}}\left[\Lambda \Psi^{n_{1}}\right]\right\}
$$

where we have used (3.4). Identifying $\left(n_{1}, n_{2}\right)$ with $(m, n-1)$ we see that this exactly cancels the third term in (3.8). This establishes gauge invariance of the action.

It is also easy to verify that if we use the Siegel gauge fixing $\left(b_{0}+\bar{b}_{0}\right)|\Psi\rangle=0$, then the tree level amplitude computed from this action produces the full off-shell amplitude given by

$$
\sum_{g} g_{s}{ }^{2 g-2} \int_{\mathcal{S}_{g, n}} \Omega_{6 g-6+2 n}^{(g, n)}\left(\left|\Phi_{1}\right\rangle, \cdots\left|\Phi_{n}\right\rangle\right)
$$

Essentially joining two or more interaction vertices by a Siegel gauge propagator corresponds to joining the corresponding Riemann surfaces by the plumbing fixture relation (2.8). The sum of all tree level graphs then produces the union of all integration 
cycles that can be obtained either from 1PI surfaces of genus $g$ with $n$ punctures, or by gluing 1PI surfaces of lower genera / lower number of punctures using plumbing fixture to produce a genus $g$ Riemann surface with $n$ punctures. By construction this produces all of $\mathcal{S}_{g, n}$.

Note that if we relax the condition that $|\Psi\rangle$ carries ghost number 2 and allow it to carry arbitrary ghost number, we shall obtain the Batalin-Vilkovisky (BV) master action [47]. However this will satisfy the classical master equation instead of the quantum master equation. This is in consonance with the fact that we are supposed to compute only tree amplitudes using this action. However since in computation of tree diagrams only ghost number two states appear even as intermediate states, the extra fields in this master action do not seem to play any useful role here.

\subsection{Equations of motion and the vacuum}

The equations of motion derived from the action are given by

$$
Q_{B}|\Psi\rangle+\sum_{n=1}^{\infty} \frac{1}{(n-1) !}\left[\Psi^{n-1}\right]=0 .
$$

Note the constant term arising from the $n=1$ term. This is related to the presence of the linear term proportional to $\{\Psi\}$ in the 1PI effective action from one loop onwards. Thus $|\Psi\rangle=0$ is not a classical solution, and we have to solve the classical equations of motion derived from the 1PI action to find the correct ground state. This can be done by looking for a solution to these equations iteratively as a power series in $g_{s}$, starting with $|\Psi\rangle=0$ as the leading solution at order $g_{s}{ }^{0}$. Thus if $\left|\Psi_{k}\right\rangle$ denotes the solution to order $g_{s}{ }^{k}$ then we can write the equation as $^{4}$

$$
Q_{B}\left|\Psi_{k+1}\right\rangle=-\sum_{n=1}^{\infty} \frac{1}{(n-1) !}\left[\Psi_{k}^{n-1}\right]+\mathcal{O}\left(g_{s}{ }^{k+2}\right),
$$

and solve this iteratively. Since the expansion of $\left|\Psi_{k}\right\rangle$ begins at order $g_{s}$, the sum over $n$ on the right hand side can be truncated at $n \leq k+2$, but we shall keep the upper limit to be $\infty$ for the ease of manipulating the terms. In order to ensure that there is no obstruction to this procedure we have to ensure that the right hand side is a BRST trivial state to order $g_{s}{ }^{k+1}$. A necessary condition for this is that the right hand side is BRST invariant, i.e.

$$
Q_{B} \sum_{n=1}^{\infty} \frac{1}{(n-1) !}\left[\Psi_{k}^{n-1}\right]
$$

vanishes to order $g_{s}{ }^{k+1}$. This is known to be true since the early days of string field theory (see e.g. [54]), but we shall briefly describe the proof for completeness. Using (3.5) we can express (3.15) as

$$
-\sum_{n=2}^{\infty} \frac{1}{(n-2) !}\left[\Psi_{k}^{n-2}\left(Q_{B} \Psi_{k}\right)\right]-\sum_{n=1}^{\infty} \frac{1}{(n-1) !} \sum_{\substack{n_{1}, n_{2} \geq 0 \\ n_{1}+n_{2}=n-1}} \frac{(n-1) !}{n_{1} ! n_{2} !}\left[\Psi_{k}^{n_{1}}\left[\Psi_{k}^{n_{2}}\right]\right] .
$$

\footnotetext{
${ }^{4}$ Even though the natural loop expansion parameter is $g_{s}{ }^{2}$, in some special cases, e.g. the one discussed in section 4.4, the classical solution $|\Psi\rangle$ begins its expansion at order $g_{s}$. For this reason we have taken $g_{s}$ as the expansion parameter.
} 
In the first term we can substitute for $Q_{B} \Psi_{k}$ using (3.14) with $k$ replaced by $k-1$ on the left hand side, but on the right hand side we can continue to use $\Psi_{k}$ since the net error we shall make by this in (3.16) will be of order $g_{s}^{k+2}$. The second term can be simplified by taking $n_{1}$ and $n_{2}$ to be independent summation variables. This reduces (3.16) to

$$
\sum_{n=2}^{\infty} \frac{1}{(n-2) !} \sum_{m=1}^{\infty} \frac{1}{(m-1) !}\left[\Psi_{k}^{n-2}\left[\Psi_{k}^{m-1}\right]\right]-\sum_{n_{1}, n_{2} \geq 0} \frac{1}{n_{1} ! n_{2} !}\left[\Psi_{k}^{n_{1}}\left[\Psi_{k}^{n_{2}}\right]\right]+\mathcal{O}\left(g_{s}{ }^{k+2}\right) .
$$

After identifying $n_{1}$ with $n-2$ and $n_{2}$ with $m-1$ these two terms cancel showing that (3.15) vanishes to order $g_{s}^{k+1}$.

This in turn allows us to write down an explicit solution of (3.14) in the $L_{0}^{+} \neq 0$ sector. Denoting by $\mathbf{P}$ the projection operator into zero momentum $L_{0}^{+}=0$ states and using $\left\{Q_{B}, b_{0}^{+}\right\}=L_{0}^{+}$, we can write the iterative solution to (3.14) as

$$
\left|\Psi_{k+1}\right\rangle=-\frac{b_{0}^{+}}{L_{0}^{+}} \sum_{n=1}^{\infty} \frac{1}{(n-1) !}(1-\mathbf{P})\left[\Psi_{k}^{n-1}\right]+Q_{B}\left|s_{k+1}\right\rangle+\left|\Phi_{k+1}\right\rangle,
$$

where $\left|s_{k+1}\right\rangle$ denotes the expansion of some arbitrary ghost number one, zero momentum state $|s\rangle \in \mathcal{H}_{0}$ to order $g_{s}{ }^{k+1}$ and $\left|\Phi_{k+1}\right\rangle$ satisfies

$$
\mathbf{P}\left|\Phi_{k+1}\right\rangle=\left|\Phi_{k+1}\right\rangle, \quad Q_{B}\left|\Phi_{k+1}\right\rangle=-\sum_{n=1}^{\infty} \frac{1}{(n-1) !} \mathbf{P}\left[\Psi_{k}^{n-1}\right]+\mathcal{O}\left(g_{s}{ }^{k+2}\right) .
$$

The existence of the solutions to the second equation is not automatic. To see this let us suppose that $\left|V_{i}\right\rangle$ for $i=1, \cdots N$ is a basis of states in $\mathcal{H}_{0}$ describing non-trivial elements of the BRST cohomology and carrying zero momentum, ghost number 2 and $L_{0}^{+}=0$. These in fact represent zero momentum massless states. Then in order for the second equation in (3.19) to have solution, we need

$$
\left\langle V_{i}\left|c_{0}^{-}\right| \sum_{n=1}^{\infty} \frac{1}{(n-1) !}\left[\Psi_{k}^{n-1}\right]\right\rangle=\mathcal{O}\left(g_{s}{ }^{k+2}\right) \quad \text { for } 1 \leq i \leq N .
$$

Unless this holds at each order in $g_{s}$, we cannot solve (3.14) by the iterative procedure we have described. As we shall discuss shortly, this will be related to the possible existence of massless tadpoles in the theory.

Before we proceed let us point out that during the process of carrying out this iterative procedure we shall encounter repeated operation of $\left(L_{0}^{+}\right)^{-1}(1-\mathbf{P})$ and $[\cdots]$, e.g. $\left[\cdots\left(L_{0}^{+}\right)^{-1}(1-\mathbf{P})[\cdots]\right]$. Representing $1 / L_{0}^{+}$as $\int_{0}^{\infty} d s e^{-s L_{0}^{+}}$and the $L_{0}^{-}=0$ projector as $\int d \theta e^{i \theta L_{0}^{-}}$we can interpret terms with repeated application of $1 / L_{0}^{+}$and [ ] in terms of correlation functions on a single Riemann surface obtained by joining 1PI Riemann surfaces by the plumbing fixture relations $(2.8)$. The $(1-\mathbf{P})$ factor plays a crucial role: it subtracts off the contribution from the massless states in the propagator, thereby removing possible divergences from the $s \rightarrow \infty$ end of the integral. As a result this procedure does not require any infrared regulator.

Let us now turn to another consequence of the presence of non-trivial elements of BRST cohomology in the $L_{0}^{+}=0$ sector. If eq. (3.20) holds then (3.19) has solutions. Let 
$\left|\Phi_{k+1}^{(0)}\right\rangle$ be a particular solution which differs from $\left|\Phi_{k}\right\rangle$ by terms of order $g_{s}{ }^{k+1}$. However from this we can construct a more general family of solutions to (3.19) with the same property by taking

$$
\left|\Phi_{k+1}\right\rangle=\left|\Phi_{k+1}^{(0)}\right\rangle+g_{s}^{k+1} \sum_{i} c_{i}^{(k+1)}\left|V_{i}\right\rangle
$$

where $c_{i}^{(k+1)}$ are arbitrary constants. This freedom exists at every order in $g_{s}$. However since the choice of $c_{i}^{(\ell)}$ 's for a given $\ell$ affects the solution at higher order via the recursion relations, it may so happen that (3.20) fails to hold for arbitrary choice of $c_{i}^{(\ell)}$ 's at lower order. In that case we have to adjust the $c_{i}^{(\ell)}$ 's at lower order to make (3.20) hold. If this is not possible, i.e. we cannot make (3.20) hold for any choice of $c_{i}^{(\ell)}$, s then we have to conclude that the system does not have a perturbative ground state. This is what happens if there are tadpoles of classical moduli fields in the theory. For theories in which (3.20) can be made to hold, we can divide the massless states into two kinds. For some states the constants $c_{i}^{(\ell)}$ remain undetermined at every order in $g_{s}$. These represent scalar fields without potential - the massless moduli fields. For other states the constants $c_{i}^{(\ell)}$ get fixed by requiring (3.20) to hold. These are scalar fields with potential. ${ }^{5}$

Note that even if we ignore the freedom provided by the vacuum expectation values of the moduli fields, the solution is ambiguous due the freedom of adding BRST trivial state $Q_{B}\left|s_{k+1}\right\rangle$ in (3.18). Any such term added at a given order will of course affect the higher order terms in the solution. It can be shown that this freedom reflects the freedom of transforming a given solution by a gauge transformation of the form described in (3.7). Since this is gauge equivalent to the original solution, we can set $\left|s_{k+1}\right\rangle$ to zero from the beginning.

Once we have obtained a classical solution - which we shall denote by $\Psi_{c l}$ - we can expand the action around the new background and obtain the new action. After throwing away the additive constant $S\left(\left|\Psi_{c l}\right\rangle\right)$, the new action can be expressed in terms of the fluctuation $\widehat{\Psi}=\Psi-\Psi_{c l}$ as $[48]$

$$
g_{s}-2\left[\frac{1}{2}\left\langle\widehat{\Psi}\left|c_{0}^{-} Q_{B}\right| \widehat{\Psi}\right\rangle+\sum_{n=2}^{\infty} \frac{1}{n !}\left\{\widehat{\Psi}^{n}\right\}^{\prime}\right],
$$

where we define

$$
\begin{aligned}
& \left\{A_{1} \cdots A_{k}\right\}^{\prime} \equiv \sum_{n=0}^{\infty} \frac{1}{n !}\left\{\Psi_{c l}^{n} A_{1} \cdots A_{k}\right\}, \quad \text { for } k \geq 2, \\
& {\left[A_{1} \cdots A_{k}\right]^{\prime} \equiv \sum_{n=0}^{\infty} \frac{1}{n !}\left[\Psi_{c l}^{n} A_{1} \cdots A_{k}\right], \quad \text { for } k \geq 1,} \\
& \left\{A_{1}\right\}^{\prime} \equiv 0, \quad[]^{\prime} \equiv 0 .
\end{aligned}
$$

\footnotetext{
${ }^{5}$ Most often massless scalars which are not moduli fields transform in the non-trivial representation of some gauge group and hence we can get a solution by setting them to zero from the beginning. However, as we shall discuss in section 4.4, there can some time be more than one possible choices of the constants $c_{i}^{(\ell)}$, at least in low orders in $g_{s}$, signalling the existence of multiple perturbative vacuum. In this case the $c_{i}^{(\ell)}=0$ solution may not represent the true ground state, i.e. (3.20) at higher order may force us to choose the $c_{i}^{(\ell)} \neq 0$ solution.
} 
Note the absence of linear term in $\widehat{\Psi}$ in the action signalling that $|\widehat{\Psi}\rangle=0$ is a solution of the equations of motion. Furthermore one can show, using the equations of motion satisfied by $\left|\Psi_{c l}\right\rangle$, that eqs. (3.2)-(3.5) hold with $\{\cdots\},[\cdots]$ replaced by $\{\cdots\}^{\prime},[\cdots]^{\prime}$. Thus the action $(3.22)$ has gauge invariance with $[\cdots]$ replaced by $[\cdots]^{\prime}$ in the gauge transformation laws (3.7).

Due to the presence of the $\left\{\widehat{\Psi}^{2}\right\}^{\prime}$ term in the action (3.22) the quadratic term is not controlled only by $Q_{B}$. It is convenient to introduce a new operator $\widehat{Q}_{B}$ defined via

$$
\widehat{Q}_{B}|A\rangle \equiv Q_{B}|A\rangle+[A]^{\prime}=Q_{B}|A\rangle+\sum_{n=0}^{\infty} \frac{1}{n !}\left[\Psi_{c l}^{n} A\right] .
$$

If we also define

$$
\begin{aligned}
& \left\{A_{1} \cdots A_{k}\right\}^{\prime \prime} \equiv\left\{A_{1} \cdots A_{k}\right\}^{\prime} \quad \text { for } k \geq 3, \quad\left[A_{1} \cdots A_{k}\right]^{\prime \prime} \equiv\left[A_{1} \cdots A_{k}\right]^{\prime} \quad \text { for } k \geq 2 \\
& \left\{A_{1}\right\}^{\prime \prime} \equiv 0, \quad\left\{A_{1} A_{2}\right\}^{\prime \prime} \equiv 0, \quad[]^{\prime \prime} \equiv 0, \quad\left[A_{1}\right]^{\prime \prime} \equiv 0,
\end{aligned}
$$

then one can express the action (3.22) as

$$
g_{s}^{-2}\left[\frac{1}{2}\left\langle\widehat{\Psi}\left|c_{0}^{-} \widehat{Q}_{B}\right| \widehat{\Psi}\right\rangle+\sum_{n=3}^{\infty} \frac{1}{n !}\left\{\widehat{\Psi}^{n}\right\}^{\prime \prime}\right]
$$

so that there is no linear term and the quadratic term is controlled by $\widehat{Q}_{B}$. Furthermore one can show, using the equations of motion satisfied by $\left|\Psi_{c l}\right\rangle$, that $\widehat{Q}_{B}$ is nilpotent and that eqs. (3.2)-(3.5) hold with $Q_{B},\{\cdots\},[\cdots]$ replaced by $\widehat{Q}_{B},\{\cdots\}^{\prime \prime},[\cdots]^{\prime \prime}$. Thus the action (3.26) has gauge invariance with $Q_{B}$ and $[\cdots]$ replaced by $\widehat{Q}_{B}$ and $[\cdots]^{\prime \prime}$ in the gauge transformation laws (3.7). This generalizes the result of [48] for classical string field theory.

Before concluding this section we shall compare the procedure described above of shifting $\Psi$ by $\Psi_{c l}$ with the conventional approach to superstring perturbation theory where no such shift is needed. As we have seen, the effect of shifting the background by $\left|\Psi_{c l}\right\rangle$ is to replace \{\} by \{\}$^{\prime}$. If in (3.18) we had dropped the second and the third terms on the right hand side, and also the projector $(1-\mathbf{P})$ from the first term, and used the corresponding expression for $\left|\Psi_{c l}\right\rangle$ to define \{\}$^{\prime}$, then this would effectively correspond to including in the definition of 1PI amplitudes also 1PR contributions where the internal propagators (i.e. punctures involved in the plumbing fixture) carry zero momentum. By replacing $\left(L_{0}^{+}\right)^{-1}$ by $\int_{0}^{\infty} d s e^{-s L_{0}^{+}}$we can interpret these as the 'tadpole diagrams' of conventional perturbation theory. However in that case one needs to use suitable upper cut-off $\Lambda$ on the $s$ integral at the intermediate stage of the calculation to tame the divergences coming from the $L_{0}^{+}=0$ states [33]. One then has to check that at the end of the calculation we can take the cut-off to infinity without encountering any divergence. In the approach described above, where we use the full expression (3.18), we do not need any infrared regulator since effectively we subtract the contribution from $L_{0}^{+}=0$ states from $\int_{0}^{\infty} d s e^{-s L_{0}^{+}}$, and then take into account the contribution from these missing states separately. However in this approach we need to ensure that at each order in $g_{s}$ we can find a solution to (3.19) that can be used for constructing the solution to the next order. This is equivalent to checking the absence of tadpole divergence in conventional perturbation theory. 


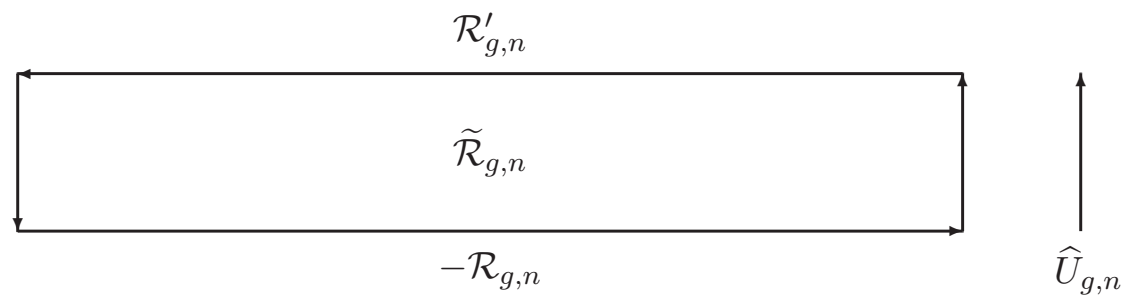

Figure 1. A pictorial representation of eqs. (3.27) and (3.28). The right hand side of (3.27) which is also the left hand side of (3.28) is the contribution to the boundary integral of $\Omega_{6 g-6+2 n}^{(g, n)}$ from the upper and lower horizontal edges of the rectangle. The first term on the right hand side of (3.28) is the volume integral of $d \Omega_{6 g-6+2 n}^{(g, n)}$ over the interior $\widetilde{\mathcal{R}}_{g, n}$ of the rectangle. Since the height of the rectangle is infinitesimal we can replace the effect of integration along the vertical direction by contraction with $\widehat{U}_{g, n}$. Finally the last term of (3.28) represents the negative of the contribution to the boundary integral of $\Omega_{6 g-6+2 n}^{(g, n)}$ from the vertical edges of the rectangle. Thus (3.28) follows from Stoke's theorem. Although we have taken the height of the rectangle to be constant for the ease of drawing the figure, this is certainly not necessary. Finally note that here we have drawn $\mathcal{R}_{g, n}$ and $\mathcal{R}_{g, n}^{\prime}$ as one dimensional horizontal lines, but the general case corresponds to them being multidimensional, with the whole figure stretching out of the plane of the paper / screen.

\subsection{Effect of changing the local coordinates and/or PCO locations}

We now turn to the problem of studying the effect of changing the choice of local coordinates and/or the locations of the PCO's on the 1PI action. A change of this form will correspond to a new choice of the $(6 g-6+2 n)$ dimensional regions $\mathcal{R}_{g, n}$ in $\widetilde{\mathcal{P}}_{g, n}$ satisfying $(2.9)$. Let us denote them by $\mathcal{R}_{g, n}^{\prime}$. We shall consider infinitesimal deformations so that $\mathcal{R}_{g, n}$ and $\mathcal{R}_{g, n}^{\prime}$ are close in $\widetilde{\mathcal{P}}_{g, n}$. Then we can write

$$
\delta S=\sum_{g=0}^{\infty} g_{s}^{2 g-2} \sum_{n=1}^{\infty} \frac{1}{n !}\left[\left(\int_{\mathcal{R}_{g, n}^{\prime}}-\int_{\mathcal{R}_{g, n}}\right) \Omega_{6 g-6+2 n}^{(g, n)}\left(|\Psi\rangle^{\otimes n}\right)\right]
$$

where $|\Psi\rangle^{\otimes n}$ denotes that there are $n$ entries of $|\Psi\rangle$ in the argument. Let $\hat{U}_{g, n}$ be an infinitesimal vector field that takes a point in $\mathcal{R}_{g, n}$ to a neighbouring point in $\mathcal{R}_{g, n}^{\prime}$. The definition of $\hat{U}_{g, n}$ is ambiguous up to addition of infinitesimal tangent vectors of $\mathcal{R}_{g, n}$, but this will not affect the final result. In this case (3.27) can be expressed as [49]

$$
\delta S=\sum_{g=0}^{\infty} g_{s}^{2 g-2} \sum_{n=1}^{\infty} \frac{1}{n !}\left[\int_{\mathcal{R}_{g, n}} d \Omega_{6 g-6+2 n}^{(g, n)}\left[\hat{U}_{g, n}\right]\left(|\Psi\rangle^{\otimes n}\right)+\int_{\partial \mathcal{R}_{g, n}} \Omega_{6 g-6+2 n}^{(g, n)}\left[\hat{U}_{g, n}\right]\left(|\Psi\rangle^{\otimes n}\right)\right]
$$

where for any $p$-form $\omega_{p}, \omega_{p}[\hat{U}]$ denotes the contraction of $\omega_{p}$ with the vector field $\hat{U}$ :

$$
\omega_{i_{1} \cdots i_{p}} d y^{i_{1}} \wedge \cdots \wedge d y^{i_{p}}[\widehat{U}] \equiv \widehat{U}^{i_{1}} \omega_{i_{1} i_{2} \cdots i_{p}} d y^{i_{2}} \wedge \cdots \wedge d y^{i_{p}}
$$

Intuitively this equation can be understood as follows. The first term on the right hand side represents the integral of $d \Omega_{6 g-6+2 n}^{(g, n)}$ over a $6 g-5+2 n$ dimensional region $\widetilde{\mathcal{R}}_{g, n}$ bounded by $\mathcal{R}_{g, n}$ and $\mathcal{R}_{g, n}^{\prime}$. This can be integrated to give (3.27) together with a contribution from the component of the boundary of $\widetilde{\mathcal{R}}_{g, n}$ that joins $\partial \mathcal{R}_{g, n}^{\prime}$ to $\partial \mathcal{R}_{g, n}$. The second term in (3.28) subtracts this contribution. A pictorial representation of this can be found in figure 1 . 
We shall now show following [49] that the change in action given in (3.28) can be regarded as the result of a redefinition of the field $|\Psi\rangle$ to $|\Psi\rangle+|\tilde{\delta} \Psi\rangle$ where $|\tilde{\delta} \Psi\rangle$ is given by

$$
\left\langle\Phi\left|c_{0}^{-}\right| \tilde{\delta} \Psi\right\rangle=-\sum_{g=0}^{\infty} g_{s}{ }^{2 g} \sum_{n=1}^{\infty} \frac{1}{(n-1) !} \int_{\mathcal{R}_{g, n}} \Omega_{6 g-5+2 n}^{(g, n)}\left[\hat{U}_{g, n}\right]\left(|\Phi\rangle,|\Psi\rangle^{\otimes(n-1)}\right),
$$

for any state $|\Phi\rangle$ in $\mathcal{H}_{0}$. We now see that since this is integrated over $\mathcal{R}_{g, n}$, adding a tangent vector of $\mathcal{R}_{g, n}$ to $\hat{U}_{g, n}$ will not change the integral. To prove (3.30), let us denote by $\tilde{\delta} S$ the change in the action induced by the field redefinition (3.30). Then from (3.6) we get

$$
\tilde{\delta} S=g_{s}{ }^{-2}\left[\left\langle\Psi\left|c_{0}^{-} Q_{B}\right| \tilde{\delta} \Psi\right\rangle+\sum_{n=1}^{\infty} \frac{1}{(n-1) !}\left\{\Psi^{n-1} \tilde{\delta} \Psi\right\}\right] .
$$

The first term can be written as

$$
\begin{aligned}
g_{s}^{-2}\left\langle Q_{B} \Psi\left|c_{0}^{-}\right| \tilde{\delta} \Psi\right\rangle & =-\sum_{g=0}^{\infty} g_{s}{ }^{2 g-2} \sum_{n=1}^{\infty} \frac{1}{(n-1) !} \int_{\mathcal{R}_{g, n}} \Omega_{6 g-5+2 n}^{(g, n)}\left[\hat{U}_{g, n}\right]\left(Q_{B}|\Psi\rangle,|\Psi\rangle^{\otimes(n-1)}\right) \\
& =\sum_{g=0}^{\infty} g_{s}{ }^{2 g-2} \sum_{n=1}^{\infty} \frac{1}{n !} \int_{\mathcal{R}_{g, n}} d \Omega_{6 g-6+2 n}^{(g, n)}\left[\hat{U}_{g, n}\right]\left(|\Psi\rangle^{\otimes n}\right)
\end{aligned}
$$

In going from the second to the final expression in the above equation we first averaged over all the $n$ possible position of $Q_{B}|\Psi\rangle$ inside the argument of $\Omega_{6 g-5+2 n}^{(g, n)}\left[\hat{U}_{g, n}\right]$, and then used (2.6). This agrees with the first term on the right hand side of (3.28). Thus it remains to show that the second term on the right hand side of (3.31) agrees with the second term on the right hand side of (3.28). Using (2.9) the latter can be expressed as

$$
-\frac{1}{2} \sum_{g=0}^{\infty} g_{s}^{2 g-2} \sum_{n=0}^{\infty} \frac{1}{n !} \sum_{\substack{g_{1}, g_{2}, n_{1}, n_{2} \\ g_{1}+g_{2}=g, n_{1}+n_{2}=n+2}} \int_{\mathbf{S}\left[\left\{\mathcal{R}_{g_{1}, n_{1}}, \mathcal{R}_{g_{2}, n_{2}}\right\}\right]} \Omega_{6 g-6+2 n}^{(g, n)}\left[\hat{U}_{g, n}\right]\left(|\Psi\rangle^{\otimes n}\right),
$$

up to an additive constant. The additive constant corresponds to the $n=0$ term in (3.33) and has no effect on any physical quantity. We can now carry out the integral over the angular variable $\theta$ in the plumbing fixture relation (2.8) and use the relation (2.10). On $\left\{\mathcal{R}_{g_{1}, n_{1}}, \mathcal{R}_{g_{2}, n_{2}}\right\}$ the infinitesimal vector field $\hat{U}_{g, n}$ can be written as a sum of two vector fields - one with support on $\mathcal{R}_{g_{1}, n_{1}}$, characterizing the difference between $\mathcal{R}_{g_{1} . n_{1}}^{\prime}$ and $\mathcal{R}_{g_{1}, n_{1}}$ and the other with support on $\mathcal{R}_{g_{2}, n_{2}}$, characterizing the difference between $\mathcal{R}_{g_{2}, n_{2}}^{\prime}$ and $\mathcal{R}_{g_{2}, n_{2}}$. Now since (3.33) is invariant under $\left(g_{1}, n_{1}\right) \leftrightarrow\left(g_{2}, n_{2}\right)$, the two terms give the same contribution. Thus we can keep only one of the terms, e.g. where $\hat{U}_{g, n}$ has support on $\mathcal{R}_{g_{2}, n_{2}}$ and multiply the result by a factor of 2 . This allows us to write (3.33) as

$$
\begin{aligned}
& -\sum_{g=0}^{\infty} g_{s}{ }^{2 g-2} \sum_{n=0}^{\infty} \frac{1}{n !} \sum_{\substack{g_{1}, g_{2}, n_{1}, n_{2} \\
g_{1}+g_{2}=g, n_{1}+n_{2}=n+2}} \mathbf{S}\left[\int_{\mathcal{R}_{g_{1}, n_{1}}} \Omega_{6 g_{1}-6+2 n_{1}}^{\left(g_{1}, n_{1}\right)}\left(|\Psi\rangle^{\otimes\left(n_{1}-1\right)},\left|\varphi_{r}\right\rangle\right)\right. \\
& \left.\int_{\mathcal{R}_{g_{2}, n_{2}}} \Omega_{6 g_{2}-5+2 n_{2}}^{\left(g_{2}, n_{2}\right)}\left[\hat{U}_{g_{2}, n_{2}}\right]\left(\left|\varphi^{r}\right\rangle,|\Psi\rangle^{\otimes\left(n_{2}-1\right)}\right)\right]
\end{aligned}
$$


In arriving at (3.34) we have taken into account the fact that besides the sign given in (2.10), there is an extra minus sign resulting from the fact that the operation of contracting a form with $\theta$ independent vector field $\widehat{U}$ with vanishing $\widehat{U}^{\theta}$ and integrating the form over $\theta$ anticommute, e.g.

$$
\int_{\theta}(d y \wedge d \theta[\widehat{U}])=\widehat{U}^{y} \int_{\theta} d \theta=2 \pi \widehat{U}^{y}, \quad\left(\int_{\theta} d y \wedge d \theta\right)[\widehat{U}]=-2 \pi d y[\widehat{U}]=-2 \pi \widehat{U}^{y} .
$$

Now since all the external states are $|\Psi\rangle$, the $\mathbf{S}$ operation in (3.34) just produces a factor of $n ! /\left(n_{1}-1\right) !\left(n_{2}-1\right)$ !. Regarding $\left(g_{1}, n_{1}\right)$ and $\left(g_{2}, n_{2}\right)$ as independent summation variables and performing the sum over $g_{1}$, we can express (3.34) as

$$
\begin{aligned}
& \left.-g_{s}{ }^{-2} \sum_{n_{1}=1}^{\infty} \frac{1}{\left(n_{1}-1\right) !}\left\{\Psi^{n_{1}-1} \varphi_{r}\right\} \sum_{g_{2}=0}^{\infty} \sum_{n_{2}=1}^{\infty} \frac{1}{\left(n_{2}-1\right) !} g_{s}^{2 g_{2}} \int_{\mathcal{R}_{g_{2}, n_{2}}} \Omega_{6 g_{2}-5+2 n_{2}}^{\left(g_{2}, n_{2}\right)}\left[\hat{U}_{g_{2}, n_{2}}\right]\left(\left|\varphi^{r}\right\rangle,|\Psi\rangle\right\rangle^{\otimes\left(n_{2}-1\right)}\right) \\
& =g_{s}{ }^{-2} \sum_{n_{1}=1}^{\infty} \frac{1}{\left(n_{1}-1\right) !}\left\{\Psi^{n_{1}-1} \varphi_{r}\right\}\left\langle\varphi^{r}\left|c_{0}^{-}\right| \tilde{\delta} \Psi\right\rangle=g_{s}{ }^{-2} \sum_{n_{1}=1}^{\infty} \frac{1}{\left(n_{1}-1\right) !}\left\{\Psi^{n_{1}-1} \tilde{\delta} \Psi\right\} .
\end{aligned}
$$

In the last step we have used the relation $\left|\varphi_{r}\right\rangle\left\langle\varphi^{r}\left|c_{0}^{-}\right| s\right\rangle=|s\rangle$ for $|s\rangle \in \mathcal{H}_{0}$ - this follows from (2.11). (3.36) agrees with the second term on the right hand side of (3.31), establishing the equality of (3.28) and (3.31) up to an additive constant.

Finally we note that even though we have described the field redefinition in terms of the original string field $\Psi$, we can also translate this to a redefinition of the shifted field $\widehat{\Psi}$ using the known relation between $\widehat{\Psi}$ and $\Psi$.

The result of this subsection and the previous one leads to the following question. Suppose that we have two different choices of $\mathcal{R}_{g, n}$ leading to two different actions. Suppose further that for each action we have constructed vacuum solutions following the procedure described in section 3.3. If we now perform the field redefinition described in this subsection to relate the two actions, does it map these vacuum solutions to each other? If the vacuum solutions are unique up to gauge transformations, then it is guaranteed that they will be mapped to each other up to a gauge transformation. However if there are moduli fields whose vacuum expectation values are not fixed, then all that one can conclude is that the family of vacuum solutions for the two actions will be related to each other under field redefinition, but the transformation rules for the parameters $c_{i}^{(n)}$ in (3.21) under this transformation will be very complicated in general. In particular even if we take all the $c_{i}^{(n)}$ 's to be zero while solving the equations of motion derived from the first action, it may not map to the solution with vanishing $c_{i}^{(n)}$ 's in the family of solutions to the equations of motion derived from the second action. This explains the observation of $[18,33,42]$ that a change in the local coordinate system and/or PCO locations will have to be accompanied by a shift in the expectation values of the moduli fields in order to ensure that we are in the same physical theory, i.e. to keep the mass spectrum and S-matrix elements unchanged.

\section{Applications}

We shall now describe how the 1PI effective action can be used to simplify the analysis of mass renormalization and vacuum shift in string theory. It should be kept in mind however 
that since the 1PI effective action gives the same off-shell amplitudes as those obtained using the prescription used in [15-18], the results remain unchanged. Only the proof of some of the results, in particular the result that physical quantities are independent of the choice of local coordinates and PCO locations used to define the off-shell amplitudes, simplify.

\subsection{Mass renormalization}

We begin our discussion with the computation of the renormalized mass. Once we have found the vacuum, the 1PI action expanded around the vacuum has no linear term. The quadratic terms define the kinetic operator $M$. If we expand the string field in some basis $\left\{\left|\bar{\varphi}_{r}\right\rangle\right\}$ of ghost number two states in $\mathcal{H}_{0}$, then the explicit form of $M_{r s}$ is given by

$$
M_{r s}=\left\langle\bar{\varphi}_{r}\left|c_{0}^{-} \widehat{Q}_{B}\right| \bar{\varphi}_{s}\right\rangle,
$$

where $\widehat{Q}_{B}$ has been defined in (3.24). Now we examine the eigenvalues of $M{ }^{6}$ This can be done at fixed momentum along the non-compact directions due to translational invariance along those directions, and then we can study how the eigenvalues vary as a function of the momentum. There are three kinds of behaviour:

1. Some of the eigenvalues will vanish for all momenta. These are pure gauge directions.

2. Some eigenvalues never vanish as a function of momentum. These are unphysical modes.

3. Some eigenvalues vary as a function of momentum $k$ and vanish at specific values of $k^{2}$. These describe physical states. The locations of the zeroes in the $-k^{2}$ plane give the physical renormalized mass ${ }^{2}$.

As an example from field theory, we can consider the free photon kinetic term in quantum electrodynamics. In momentum space it is proportional to $\left(-k^{2} \eta^{\mu \nu}+k^{\mu} k^{\nu}\right)$. Taking $\left\{k^{\mu}\right\}=\left(k^{0}, k^{1}, 0,0\right)$ the kinetic operator takes the form

$$
\left(\begin{array}{cccc}
\left(k^{1}\right)^{2} & k^{0} k^{1} & & \\
k^{0} k^{1} & \left(k^{0}\right)^{2} & & \\
& & \left(k^{0}\right)^{2}-\left(k^{1}\right)^{2} & \\
& & & \left(k^{0}\right)^{2}-\left(k^{1}\right)^{2}
\end{array}\right)
$$

This has eigenvalues

$$
\lambda_{1}(k)=0, \quad \lambda_{2}(k)=\left(k^{0}\right)^{2}+\left(k^{1}\right)^{2}, \quad \lambda_{3}(k)=\lambda_{4}(k)=\left(k^{0}\right)^{2}-\left(k^{1}\right)^{2} .
$$

Since $\lambda_{1}(k)$ vanishes for all $k$, it represents a pure gauge mode. $\lambda_{2}(k)$ never vanishes (except at the special point $\left.k^{0}=k^{1}=0\right)$ and hence describes an unphysical mode. $\lambda_{3}(k)$ and $\lambda_{4}(k)$ vanish at $k^{0}=k^{1}$, and describe massless physical states. The important point to note is that the mass spectrum can be found without any gauge fixing. If instead we had added a

\footnotetext{
${ }^{6}$ Note that even though $\widehat{Q}_{B}$ is nilpotent, the matrix $M$ is not nilpotent. Hence it can have non-zero eigenvalues.
} 
gauge fixing term and then computed the eigenvalues of the kinetic term and the locations of their zeroes, the result will in general depend on the choice of gauge. We'll then have to make special effort to determine which of these zeroes describe physical states (for which the location of the zeroes in the $k^{2}$ plane should not depend on the gauge choice) and which are gauge artifacts. This was the main problem encountered in the analysis of [16]. Here we see that the use of gauge invariant 1PI effective action allows us to circumvent this problem by working with the gauge invariant quantum corrected kinetic term.

Now consider the effect of a change in the choice of local coordinates and/or PCO locations. As discussed in section 3.4, this can be compensated by a field redefinition under which the original vacuum gets mapped to a vacuum of the new action for some specific choice of the moduli fields. As a result the kinetic operator $M$ around the corresponding vacua are related to each other by a transformation of the form

$$
M \rightarrow A(-k)^{T} M A(k),
$$

where $A(k)$ is an operator acting on states of ghost number two and momentum $k$ in $\mathcal{H}_{0}$ that can be computed using the field redefinition (3.30). The important point to note is that since (3.30) involves integration over the 1PI regions $\mathcal{R}_{g, n}$, it does not have any pole in the $-k^{2}$ plane in perturbation theory. Thus for every eigenstate of zero eigenvalue of $M$, we can construct an eigenstate of zero eigenvalue of $A(-k)^{T} M A(k)$ by multiplying the original eigenstate by the non-singular matrix $A(k)^{-1}$. This shows that the locations of the zeroes of the eigenvalues in the $-k^{2}$ plane are not affected by the field redefinition. This is turn establishes that the physical renormalized masses are independent of the choice of local coordinate system and the locations of the PCO's.

The above discussion has been somewhat formal, in the sense that it requires us to work with infinite dimensional matrices $M$. We shall now describe how to reduce the problem of computing renormalized mass to a more manageable form by 'integrating out' contribution from all states except those at a given mass level $m$ - where mass level of a state carrying momentum $k$ is defined by the condition that its $L_{0}^{+}$eigenvalue vanishes for $k^{2}=-m^{2}$. This will then allow us to work with states of a given mass level at a time, which are finite in number. During this analysis we shall also develop a systematic perturbation expansion for computing the renormalized masses.

We recall that in order to address the problem of mass renormalization we have to find zero eigenvalues of $M$, i.e. find solutions to the equation

$$
\widehat{Q}_{B}|\psi\rangle=0 \text {. }
$$

Let us express $\widehat{Q}_{B}$ as

$$
\widehat{Q}_{B}=Q_{B}+K, \quad K|A\rangle \equiv \sum_{n=0}^{\infty} \frac{1}{n !}\left[\Psi_{c l}^{n} A\right]
$$

so that $K$ contains operators of order $g_{s}^{2}$ and higher. Since the natural expansion parameter is $\kappa \equiv g_{s}{ }^{2}$, we shall from now on express all the quantities as a power series expansion in $\kappa .^{7}$

\footnotetext{
${ }^{7}$ Here we are ignoring the special cases discussed in section 4.4 where $\left|\Psi_{c l}\right\rangle$ can be of order $g_{s}$ leading to $g_{s}$ as the expansion parameter. Our analysis can be extended to these cases as well.
} 
We shall denote by $\left|\psi_{n}\right\rangle$ the result for $|\psi\rangle$ accurate to order $\kappa^{n}$, and the same convention will be followed for all other states. Then we can express (4.5) as

$$
Q_{B}\left|\psi_{n+1}\right\rangle=-K\left|\psi_{n}\right\rangle+\mathcal{O}\left(\kappa^{n+2}\right) .
$$

Let us suppose that we have found $\left|\psi_{n}\right\rangle$ satisfying (4.7) with $n$ replaced by $(n-1)$. Then using the nilpotence of $Q_{B}+K$ one can show that $Q_{B} K\left|\psi_{n}\right\rangle=\mathcal{O}\left(\kappa^{n+2}\right)$. We can now write down a formal solution to (4.7) of the form ${ }^{8}$

$$
\left|\psi_{n+1}\right\rangle=-\frac{b_{0}^{+}}{L_{0}^{+}} K\left|\psi_{n}\right\rangle+\mathcal{O}\left(\kappa^{n+2}\right) .
$$

(4.8) makes sense as long as $K\left|\psi_{n}\right\rangle$ is a linear combination of states with $L_{0}^{+} \neq 0$. Now while applying the above procedure we shall always begin with an initial state $\left|\psi_{0}\right\rangle$ at some given mass level $m$ and proceed. On shell condition at tree level then requires the momentum carried by the state to satisfy $k^{2}=-m^{2}$ i.e. $L_{0}^{+}=0$. Since in perturbation theory we shall keep the momentum $k$ close to the original value we see that for states at the same mass level $m$ as $\left|\psi_{0}\right\rangle, L_{0}^{+}$eigenvalues will be small (of order $\kappa$ ) and hence the operator $\frac{b_{0}^{+}}{L_{0}^{+}} K$ may be of order one, signalling a breakdown of the perturbative procedure. For this purpose we shall introduce a projection operator $P$ that projects onto states of mass level $m$ and apply this recursive technique only on states other than those at mass level $m$. Let us suppose that we want to compute the renormalized masses accurately up to order $\kappa^{N}$. Then we claim that the following is a solution to (4.7) for $n \leq N-1$

$$
\left|\psi_{0}\right\rangle=\left|\phi_{N}\right\rangle, \quad\left|\psi_{n+1}\right\rangle=-\frac{b_{0}^{+}}{L_{0}^{+}}(1-P) K\left|\psi_{n}\right\rangle+\left|\phi_{N}\right\rangle+\mathcal{O}\left(\kappa^{n+2}\right)
$$

where $\left|\phi_{N}\right\rangle$ satisfies

$$
\begin{gathered}
P\left|\phi_{N}\right\rangle=\left|\phi_{N}\right\rangle, \\
Q_{B}\left|\phi_{N}\right\rangle=-P K\left|\psi_{N-1}\right\rangle+\mathcal{O}\left(\kappa^{N+1}\right) .
\end{gathered}
$$

The proof that (4.9) satisfies (4.7) goes as follows. The projection condition (4.10) tells us that $\left|\phi_{N}\right\rangle$ is a level $m$ state. ${ }^{9}$ Using (4.9) to express $\left|\psi_{n+1}\right\rangle-\left|\psi_{n}\right\rangle$ in terms of $\left|\psi_{n}\right\rangle-\left|\psi_{n-1}\right\rangle$ and noting that $\left.\left|\psi_{1}\right\rangle-\psi_{0}\right\rangle$ is of order $\kappa$ one can show iteratively that $\left|\psi_{\ell+1}\right\rangle-\left|\psi_{\ell}\right\rangle \sim \kappa^{\ell+1}$ for all $\ell$. This observation, together with eq. (4.11), gives $Q_{B}\left|\phi_{N}\right\rangle=-P K\left|\psi_{n}\right\rangle+\mathcal{O}\left(\kappa^{n+2}\right)$. Using this and assuming that (4.7) holds with $n$ replaced by $(n-1)$ one can easily verify that (4.9) satisfies (4.7).

By iterating this solution till $n=N-1$ starting with the seed solution $\left|\psi_{0}\right\rangle=\left|\phi_{N}\right\rangle$ we can determine $\left|\psi_{n}\right\rangle$ in terms of $\left|\phi_{N}\right\rangle$ for $0 \leq n \leq N$. In particular we get an expression for $\left|\psi_{N-1}\right\rangle$ in terms of $\left|\phi_{N}\right\rangle$ of the form

$$
\left|\psi_{N-1}\right\rangle=S\left|\phi_{N}\right\rangle+\mathcal{O}\left(\kappa^{N}\right)
$$

\footnotetext{
${ }^{8} \mathrm{~A}$ more general solution to (4.7) will allow us to add a term of the form $Q_{B}\left|\chi_{n+1}\right\rangle$ to the right hand side of (4.9). We are allowed to drop such terms this since we are interested in finding solutions to (4.7) which are not pure gauge deformations.

${ }^{9}$ Notice that we have not introduced the states $\left|\phi_{n}\right\rangle$ for $0 \leq n \leq N-1$ which would provide approximations to $\left|\phi_{N}\right\rangle$ to order $\kappa^{n}$. Instead of determining $\left|\phi_{n}\right\rangle$ perturbatively, we shall later determine it in a single step.
} 
for some linear operator $S . S$ involves multiple successive operations of $(1-P)\left(L_{0}^{+}\right)^{-1}$ and $K$, and, as in section 3.3 , each term involving such products of operators may be interpreted as contribution from a single Riemann surface obtained by joining 1PI Riemann surfaces via plumbing fixture. The effect of the $(1-P)$ operator is to remove the contribution from the states of mass level $m$ from the propagator which could generate large numbers of order $1 / \kappa$ from the $s$ integral.

Eq. (4.11) now gives

$$
Q_{B}\left|\phi_{N}\right\rangle=-P K S\left|\phi_{N}\right\rangle+\mathcal{O}\left(\kappa^{N+1}\right)
$$

Since $\left|\phi_{N}\right\rangle$ is an arbitrary state of mass level $m$, we can express this as a linear combination of the basis states $|r\rangle \in \mathcal{H}_{0}$ of ghost number two and mass level $m$ :

$$
\left|\phi_{N}\right\rangle=\sum_{r} v_{r}|r\rangle
$$

Taking the inner product of (4.13) with $\langle s| c_{0}^{-}$where $\langle s|$ is the BPZ conjugate of $|s\rangle$, we get

$$
\sum_{r}\left\langle s\left|c_{0}^{-}\left(Q_{B}+P K S\right)\right| r\right\rangle v_{r}=\mathcal{O}\left(\kappa^{N+1}\right) .
$$

Thus the problem reduces to finding the zero eigenvalues of a finite dimensional matrix. Solutions which exist for all $k$ will represent pure gauge states while solutions which exist only for some fixed value of $k^{2}$ near $-m^{2}$ will represent physical states. Explicit form of pure gauge solutions will be given in (4.17), (4.18).

In the next subsection we shall describe the relationship of this approach to the Siegel gauge analysis of [16].

\subsection{Relation to Siegel gauge analysis}

Ref. [16] gave a prescription for computing mass renormalization, but it was based on the analysis of Siegel gauge propagator and hence is apparently different from the definition of physical renormalized mass we have suggested above. We shall now show that the definition given in section 4.1 agrees with the one in [16], and hence we can use the argument given in section 4.1 to conclude that the perturbative method described in [16] also gives results which are independent of the choice of local coordinate system and locations of PCO's.

Our strategy will be to show that any solution to (4.7) can be made to satisfy the Siegel gauge condition after adding to it a pure gauge state. This will justify the approach of [16] which worked with states in the Siegel gauge. First we note that by construction $\left|\psi_{n+1}\right\rangle$ given in (4.9) satisfies the Siegel gauge condition for states at level other than $m$, i.e. satisfies

$$
b_{0}^{+}(1-P)\left|\psi_{n+1}\right\rangle=\mathcal{O}\left(\kappa^{n+2}\right),
$$

Thus we need to show that $\left|\phi_{N}\right\rangle$ can also be made to satisfy Siegel gauge condition. We shall do this by exploiting the freedom of adding pure gauge solutions to $\left|\psi_{n+1}\right\rangle$, but for this we must first find the form of the pure gauge solutions satisfying (4.16). We shall now 
describe an algorithm to generate such solutions. We first construct $\left|\lambda_{n}\right\rangle$ for $0 \leq n \leq N$ by solving the recursion relations

$$
\left|\lambda_{0}\right\rangle=\left|\eta_{N}\right\rangle, \quad\left|\lambda_{n+1}\right\rangle=-\frac{b_{0}^{+}}{L_{0}^{+}}(1-P) K\left|\lambda_{n}\right\rangle+\left|\eta_{N}\right\rangle+\mathcal{O}\left(\kappa^{n+2}\right),
$$

where $\left|\eta_{N}\right\rangle$ is an arbitrary state of ghost number 1 , momentum $k$ and mass level $m$ in $\mathcal{H}_{0}$, with its $\kappa$ expansion beginning at $\kappa^{0}$ and going up to $\kappa^{N}$. Using (4.17) to express $\left|\lambda_{n+1}\right\rangle-\left|\lambda_{n}\right\rangle$ in terms of $\left|\lambda_{n}\right\rangle-\left|\lambda_{n-1}\right\rangle$ and noting that $\left|\lambda_{1}\right\rangle$ and $\left|\lambda_{0}\right\rangle$ differ by order $\kappa$, one can show iteratively that $\left|\lambda_{n+1}\right\rangle-\left|\lambda_{n}\right\rangle=\mathcal{O}\left(\kappa^{n+1}\right)$. We now define

$\left|\psi_{n+1}^{g}\right\rangle=\left(Q_{B}+K\right)\left|\lambda_{n+1}\right\rangle+\mathcal{O}\left(\kappa^{n+2}\right)=Q_{B}\left|\lambda_{n+1}\right\rangle+K\left|\lambda_{n}\right\rangle+\mathcal{O}\left(\kappa^{n+2}\right) \quad$ for $0 \leq n+1 \leq N$.

It follows using the nilpotence of $\left(Q_{B}+K\right)$ that $\left|\psi_{n+1}^{g}\right\rangle$ satisfies (4.7) at generic momentum and hence is a pure gauge state. Furthermore from (4.17) it is easy to check that $\left|\psi_{n+1}^{g}\right\rangle$ satisfies (4.16).

Let us now focus our attention on the physical states. Suppose that $m_{R}=m+\mathcal{O}(\kappa)$ is the correct renormalized mass for some physical state. Then at $k^{2}=-m_{R}{ }^{2}$ it will have the general form given in (4.9)-(4.11). Now if we add to it any pure gauge state of the from (4.18) carrying the same momentum, it will continue to remain an eigenstate of the kinetic operator with zero eigenvalue. Our goal will be to argue that by adding appropriate pure gauge states of the form (4.18) we can make each of the renormalized physical states satisfy Siegel gauge condition. Since we shall be computing the solution accurate up to order $\kappa^{N}$ we shall take a pure gauge solution up to that order. Thus the general solution takes the form

$$
\left|\chi_{N}\right\rangle \equiv\left|\psi_{N}\right\rangle+\left(Q_{B}+K\right)\left|\lambda_{N}\right\rangle=\left|\psi_{N}\right\rangle+Q_{B}\left|\lambda_{N}\right\rangle+K\left|\lambda_{N-1}\right\rangle+\mathcal{O}\left(\kappa^{N+1}\right)
$$

where $\left|\psi_{n+1}\right\rangle$ and $\left|\lambda_{n+1}\right\rangle$ satisfy the recursion relations (4.9) and (4.17) for $0 \leq n \leq(N-1)$ :

$$
\begin{aligned}
&\left|\psi_{0}\right\rangle=\left|\phi_{N}\right\rangle,\left|\psi_{n+1}\right\rangle=-\frac{b_{0}^{+}}{L_{0}^{+}}(1-P) K\left|\psi_{n}\right\rangle+\left|\phi_{N}\right\rangle+\mathcal{O}\left(\kappa^{n+2}\right), \\
&\left|\lambda_{0}\right\rangle=\left|\eta_{N}\right\rangle, \quad\left|\lambda_{n+1}\right\rangle=-\frac{b_{0}^{+}}{L_{0}^{+}}(1-P) K\left|\lambda_{n}\right\rangle+\left|\eta_{N}\right\rangle+\mathcal{O}\left(\kappa^{n+2}\right) .
\end{aligned}
$$

Using (4.19), (4.20) we now get

$$
b_{0}^{+}\left|\chi_{N}\right\rangle=b_{0}^{+}\left(\left|\phi_{N}\right\rangle+P K\left|\lambda_{N-1}\right\rangle+Q_{B}\left|\eta_{N}\right\rangle\right)+\mathcal{O}\left(\kappa^{N+1}\right) .
$$

Now it follows from (4.11) that $Q_{B}\left|\phi_{N}\right\rangle$ has its expansion starting at order $\kappa$. Thus in the $\kappa \rightarrow 0$ limit $\left|\phi_{N}\right\rangle$ is a BRST invariant state. It then follows from the general result on BRST cohomology that

$$
\left|\phi_{N}\right\rangle=c \bar{c} e^{-\phi} V(0)|0\rangle+Q_{B}|s\rangle+\kappa\left|\xi_{N-1}\right\rangle .
$$

Here $V$ is a $\kappa$ independent matter vertex operator of dimension $\left(1 / 2+\alpha^{\prime}\left(k^{2}+m^{2}\right) / 4,1+\right.$ $\left.\alpha^{\prime}\left(k^{2}+m^{2}\right) / 4\right)$ carrying momentum $k$, and becomes a superconformal primary operator in 
the $k^{2} \rightarrow-m^{2}$ limit. $|s\rangle$ is some $\kappa$ independent state of level $m$ and $\left|\xi_{N-1}\right\rangle$ is a state of level $m$ whose $\kappa$ expansion starts at order $\kappa^{0}$. Now by choosing

$$
\left|\eta_{N}\right\rangle=-|s\rangle-\frac{b_{0}^{+}}{L_{0}^{+}}\left(\kappa\left|\xi_{N-1}\right\rangle+P K\left|\lambda_{N-1}\right\rangle\right)
$$

we can make the right hand side of (4.21) vanish to order $\kappa^{N}$, showing that $\left|\chi_{N}\right\rangle$ satisfies the Siegel gauge condition. Since the $\left|\lambda_{N-1}\right\rangle$ determined from the recursion relation (4.20) is a function of $\left|\eta_{N}\right\rangle$, (4.23) gives a linear equation for $\left|\eta_{N}\right\rangle$ which needs to be solved. Furthermore this has to be done by regarding this as a matrix equation in the space of mass level $m$ states and not perturbatively, since acting on states of mass level $m, b_{0}^{+}\left(L_{0}^{+}\right)^{-1}$ is of order $1 / \kappa$ and hence each of the terms in (4.23) will have their $\kappa$ expansion beginning at order $\kappa^{0}$. For example for $N=1$ we have $\left|\lambda_{0}\right\rangle=\left|\eta_{1}\right\rangle$ and hence (4.23) takes the form

$$
\left|\eta_{1}\right\rangle=-|s\rangle-\frac{b_{0}^{+}}{L_{0}^{+}}\left(\kappa\left|\xi_{0}\right\rangle+P K\left|\eta_{1}\right\rangle\right)
$$

i.e.

$$
\left|\eta_{1}\right\rangle=-\left(1+\frac{b_{0}^{+}}{L_{0}^{+}} P K\right)^{-1}\left(|s\rangle+\kappa \frac{b_{0}^{+}}{L_{0}^{+}}\left|\xi_{0}\right\rangle\right) .
$$

Furthermore using (4.19), (4.20) we now get the leading order contribution to $\left|\chi_{N}\right\rangle$ to be

$$
\left|\chi_{N}\right\rangle=\left|\phi_{N}\right\rangle+Q_{B}\left|\eta_{N}\right\rangle+\mathcal{O}(\kappa)=c \bar{c} e^{-\phi} V(0)|0\rangle-Q_{B} \frac{b_{0}^{+}}{L_{0}^{+}}\left(P K\left|\eta_{N}\right\rangle+\kappa\left|\xi_{N-1}\right\rangle\right)+\mathcal{O}(\kappa) .
$$

This analysis shows that by adding appropriate pure gauge states we can ensure that the renormalized physical state satisfies the Siegel gauge condition at $k^{2}=-m_{R}{ }^{2}$. This in turn implies that we can, from the beginning, look for the physical states by working with states satisfying Siegel gauge condition. However this is still not equivalent to working with the Siegel gauge fixed action and studying its (linearized) equations of motion. In the latter case we not only restrict the states to be in the Siegel gauge, we also only examine a subset of the equations of motion - namely those which have components along the states in the Siegel gauge. Thus finding a zero eigenvalue of the Siegel gauge kinetic operator is a necessary but not sufficient condition for a physical state, and we need to find some additional criterion that will tell us which of the zero eigenvalues of the Siegel gauge kinetic operator describe physical states. Eq. (4.26) tells us how to identify these physical states in the Siegel gauge - in the $\kappa \rightarrow 0$ limit they must be given by a sum of a state of the form $c \bar{c} e^{-\phi} V(0)|0\rangle$ and a BRST trivial state. In the Siegel gauge analysis of [17] it was found that if at tree level there are $n_{p}$ physical states at mass level $m$, then after taking into account loop effects there are precisely $n_{p}$ states with zero eigenvalue of the kinetic operator in the Siegel gauge whose eigenstates have the form given in (4.26). There were also other zero eigenvalues whose eigenstates were not of this form. Thus the former must be the desired physical states, while the latter must be states which will fail to satisfy the linearized equations of motion when we examine its components along states outside the Siegel gauge. Indeed [17] also identified the former as the true physical states using 
a different set of arguments. The current analysis shows that these are true eigenstates with zero eigenvalue of the full kinetic operator $c_{0}^{-} \widehat{Q}_{B}$ and hence the renormalized masses associated with these eigenstates are invariant under a change of local coordinate system and / or PCO locations.

\subsection{S-matrix}

In order to compute the S-matrix from the 1PI action one needs to fix a gauge, compute the gauge fixed propagator, and then compute the tree level S-matrix in the theory using standard rules. While this does require a gauge fixing and hence introduces possible dependence on spurious data in the intermediate stage, we do not need to introduce any ghosts associated with the gauge fields since we only compute tree amplitudes. Thus the standard proof that the tree level S-matrix of a field theory is independent of the choice of gauge and field redefinition should hold. The difficult part of the proof of gauge invariance (and invariance under field redefinition) of the S-matrix elements, involving analysis of the invariance of the path integral measure under these transformations, can be avoided altogether by working with gauge invariant 1PI effective action.

\subsection{Vacuum shift}

We shall now turn to the analysis of vacuum shift studied in [16]. The set up is as follows. Suppose we have a scalar field $\phi$ that is massless at the tree level but has a tree level potential $A g_{s}{ }^{-2} \phi^{4}$ for some positive constant $A$. Suppose further that one loop correction generates a negative contribution $-B \phi^{2}$ to the potential for some positive constant $B$. Thus we have the full potential

$$
A g_{s}{ }^{-2} \phi^{4}-B \phi^{2}+\cdots
$$

where $\cdots$ denotes other terms involving higher powers of $\phi$ and/or of $g_{s}^{2}$. Now it is clear that the potential has a minimum at

$$
\phi^{2}=B g_{s}^{2} / 2 A+\cdots
$$

The question is: how do we study perturbative string theory around this minimum?

From the point of view of the 1PI effective action the answer is as follows. Since $\phi$ is massless at the tree level, one of the $c_{i}$ 's (or more generally some combination of the $c_{i}$ 's) appearing in (3.21) represents the vacuum expectation value of $\phi$. While solving the equations of motion to find $\left|\Psi_{c l}\right\rangle$, we should run into a situation where the vanishing of (3.20) can be achieved for three possible solutions corresponding to $\phi=0$ and $\phi=$ $\pm g_{s} \sqrt{B / 2 A} .{ }^{10}$ Here we need to pick the solution for $c_{i}$ 's that corresponds to the minimum of the potential at $\phi= \pm g_{s} \sqrt{B / 2 A}$ rather than the maximum at $\phi=0$. Beginning with this solution we need to systematically compute the higher order terms and correct the solution at each order to ensure the vanishing of (3.20). The actual procedure is similar

\footnotetext{
${ }^{10}$ Note that in general $\phi$ and $c_{i}$ 's will be related by complicated functions, but to order $g_{s}$ we can expect the relationship to be linear.
} 
to the one adopted in [54], although the latter paper only examined solutions to the tree level string field theory action.

Once we have found the solution to any given order we can expand the action around the minimum as in section 3.3 and carry out the usual computation of renormalized masses and S-matrix elements. The result will formally be identical to the ones described in [17]; however unlike in [17] here we shall not need to regulate the infrared divergence associated with tadpoles of $\phi$ even at the intermediate stage, since we determine the vacuum expectation value of $\phi$ (and other fields) by solving the classical equations of motion rather than demanding the vanishing of the $\phi$-tadpole. The invariance of the renormalized mass and S-matrix elements under a change of local coordinate system and/or PCO locations now follow in the same way as in section 4.1 , section 4.3 .

\section{Acknowledgments}

We thank Nathan Berkovits, Roji Pius, Arnab Rudra, Edward Witten and Barton Zwiebach for useful discussions and Barton Zwiebach for his valuable comments on an earlier version of the manuscript. This work was supported in part by the DAE project 12-R\&D-HRI-5.02-0303 and J. C. Bose fellowship of the Department of Science and Technology, India.

\section{A Issues with vertical segment}

In the analysis of [18] it was found that $\mathcal{R}_{g, n}$ 's cannot be taken to be sections over $\mathcal{K}_{g, n}$ in the strict sense - there are codimension one subspaces $\widetilde{\mathcal{K}}_{g, n}$ of $\mathcal{K}_{g, n}$ on which the locations of the PCO's will have to change from an initial configuration $\left(z_{1}, \cdots z_{p}\right)$ to a final configuration $\left(z_{1}^{\prime}, \cdots z_{p}^{\prime}\right)$ keeping the moduli fixed. In other words $\mathcal{R}_{g, n}$ contains vertical segments. Even though the path connecting $\left(z_{1}, \cdots z_{p}\right)$ to $\left(z_{1}^{\prime}, \cdots z_{p}^{\prime}\right)$ passes through the spurious singularities, there is a well defined value of the integral of $\Omega_{p}^{(g, n)}$ along this path provided the PCO locations are moved one at a time. Now we can consider two such paths in which the order in which the PCO locations are moved differ. Each of these paths describe a choice of $\mathcal{R}_{g, n}$. Let us call them $\mathcal{R}_{g, n}$ and $\mathcal{R}_{g, n}^{\prime}$. We would like to show that the actions corresponding to the choices $\mathcal{R}_{g, n}$ and $\mathcal{R}_{g, n}^{\prime}$ are related by a field redefinition. However the problem is that the change is not infinitesimal and hence we cannot directly apply the analysis of section 3.4. We shall now discuss a way to circumvent this problem.

We recall that we are allowed to choose weighted averages of different PCO prescriptions in defining the region $\mathcal{R}_{g, n}$. Thus we could take a one parameter family of regions $\mathcal{R}_{g, n}(t)$ of the form

$$
\mathcal{R}_{g, n}(t)=\sum_{i} f_{g, n}^{(i)}(t) \mathcal{R}_{g, n}^{(i)}
$$

where $\mathcal{R}_{g, n}^{(i)}$ are different choices of $\mathcal{R}_{g, n}$ differing in their vertical segments and $f_{g, n}^{(i)}(t)$ 's are appropriate weight factors labelled by a parameter $t$ satisfying

$$
\sum_{i} f_{g, n}^{(i)}(t)=1
$$


and the gluing compatibility condition

$$
\partial \mathcal{R}_{g, n}(t)=-\frac{1}{2} \sum_{\substack{g_{1}, g_{1}, n_{1}, n_{2} \\ g_{1}+g_{2}=g, n_{1}+n_{2}=n+2}} \mathbf{S}\left[\left\{\mathcal{R}_{g_{1}, n_{1}}(t), \mathcal{R}_{g_{2}, n_{2}}(t)\right\}\right]
$$

for each $t$. We choose the $f_{g, n}^{(i)}(t)$ such that at $t=0$ and 1 we get back $\mathcal{R}_{g, n}$ and $\mathcal{R}_{g, n}^{\prime}$ respectively, so that as we deform $t$ from 0 to 1 we interpolate between the two choices of the vertical segment which we wanted to show are equivalent. Now, under an infinitesimal change $\delta t$ of $t$, we get

$$
\delta \mathcal{R}_{g, n}(t)=\delta t \sum_{i} f_{g, n}^{(i) \prime}(t) \mathcal{R}_{g, n}^{(i)},
$$

where ' denotes derivative with respect to $t$. The right hand side of (A.4) is a weighted sum of subspaces of $\widetilde{\mathcal{P}}_{g, n}$. Since $\delta \mathcal{R}_{g, n}$ is infinitesimal, we might hope to apply techniques similar to those in section 3.4 to show that the change in the action can be absorbed by a field redefinition. Our goal will be to show that this can indeed be done. Once we establish this for infinitesimal deformations, the results for finite deformation will follow.

Note that we cannot take the interpolating region to be simply $(1-t) \mathcal{R}_{g, n}^{(1)}+t \mathcal{R}_{g, n}^{(2)}$ as this will violate the gluing compatibility condition (2.9). To illustrate this let us suppose that the vertical segment first appears at genus $g_{0}$ with $n_{0}$ punctures in the interior of $\mathcal{K}_{g_{0}, n_{0}}$. If $\mathcal{R}_{g_{0}, n_{0}}$ and $\mathcal{R}_{g_{0}, n_{0}}^{\prime}$ denote two such choices then we can take the interpolating integration cycles to be $\mathcal{R}_{g_{0}, n_{0}}(t)=t \mathcal{R}_{g_{0}, n_{0}}+(1-t) \mathcal{R}_{g_{0}, n_{0}}$. But now we have

$$
\left\{\mathcal{R}_{g_{0}, n_{0}}(t), \mathcal{R}_{g_{0} . n_{0}}(t)\right\}=t^{2}\left\{\mathcal{R}_{g_{0}, n_{0}}, \mathcal{R}_{g_{0} . n_{0}}\right\}+2 t(1-t)\left\{\mathcal{R}_{g_{0}, n_{0}}, \mathcal{R}_{g_{0} . n_{0}}^{\prime}\right\}+(1-t)^{2}\left\{\mathcal{R}_{g_{0}, n_{0}}^{\prime}, \mathcal{R}_{g_{0} . n_{0}}^{\prime}\right\}
$$

Since this forms a boundary of $\mathcal{R}_{2 g_{0}, 2 n_{0}-2}$ we see that the choice of the interpolating integration cycle $\mathcal{R}_{2 g_{0}, 2 n_{0}-2}(t)$ will have to be more complicated. Similarly $\mathcal{R}_{3 g_{0}, 3 n_{0}-4}(t)$, which contains $\left\{\mathcal{R}_{g_{0}, n_{0}}(t), \mathcal{R}_{2 g_{0}, 2 g_{0}}(t)\right\}$ as a boundary, must be even more complicated.

We now introduce the following (formal weighted sum of) subspaces of $\widetilde{\mathcal{P}}_{g, n}$ :

1. $\delta \mathcal{R}_{g, n}(t)$ defined in (A.4) represents a formal sum of subspaces of $\widetilde{\mathcal{P}}_{g, n}$. Due to (A.2) we have $\sum_{i} f_{g, n}^{(i) \prime}(t)=0$. Since $\mathcal{R}_{g, n}^{(i)}$ 's differ from each other only on the fiber over $\widetilde{\mathcal{K}}_{g, n}$, we see that $\delta \mathcal{R}_{g, n}(t)$ lies inside the fiber over $\widetilde{\mathcal{K}}_{g, n}$. Furthermore since the restriction of $\mathcal{R}_{g, n}^{(i)}$ to the fiber over any point $m$ in $\widetilde{\mathcal{K}}_{g, n}$ describe a path in the fiber with the same initial and final points for each $i$, the $\sum_{i} f_{g, n}^{(i) \prime}(t)=0$ relation implies that the restriction of $\delta \mathcal{R}_{g, n}(t)$ to this fiber will be a formal sum of closed paths in the fiber, or more specifically in the space of PCO locations at fixed choices of the local coordinate systems. ${ }^{11}$ We shall denote this formal sum of closed curves by $\mathcal{C}_{g, n}(m)$ for every $m \in \widetilde{\mathcal{K}}_{g, n} . \delta \mathcal{R}_{g, n}(t)$ may now be identified as the collection of $\mathcal{C}_{g, n}(m)$ 's for all $m \in \widetilde{\mathcal{K}}_{g, n}$.

${ }^{11}$ At the intersection of two such $\widetilde{\mathcal{K}}_{g, n}$ 's we have vertical segments which are two dimensional surfaces in the space of PCO locations instead of paths; on higher codimension surfaces involving intersection of multiple $\widetilde{\mathcal{K}}_{g, n}$ 's the vertical segment has even higher dimensions. There are additional subtleties involving these subspaces but they can be taken care of [51]. 
2. $\mathcal{C}_{g, n}(m)$ in turn may be regarded as the boundary of $\mathcal{D}_{g, n}(m)$ - a formal sum of two dimensional subspaces in the fiber over $m$. We can take this two dimensional subspace to lie along fixed choices of local coordinate system, letting the PCO locations vary along $\mathcal{D}_{g, n}(m)$.

3. We define $\Delta_{g, n}$ to be the formal sum of $6 g-5+2 n$ dimensional subspaces of $\widetilde{\mathcal{P}}_{g, n}$ obtained by taking the collection of the $\mathcal{D}_{g, n}(m)$ 's for all $m \in \widetilde{\mathcal{K}}_{g, n}$.

4. We also define $\Gamma_{g, n}$ to be the formal sum of $6 g-6+2 n$ dimensional subspace of $\widetilde{\mathcal{P}}_{g, n}$ obtained by taking the collection of the $\mathcal{D}_{g, n}(m)$ 's for all $m \in \partial \widetilde{\mathcal{K}}_{g, n}$. Here $\partial \widetilde{\mathcal{K}}_{g, n}$ is the boundary of $\widetilde{\mathcal{K}}_{g, n}$ given by the intersection of $\widetilde{\mathcal{K}}_{g, n}$ and $\partial \mathcal{K}_{g, n}$.

It follows from the above definitions that the boundary of $\Delta_{g, n}$ is given by

$$
\partial \Delta_{g, n}=\delta \mathcal{R}_{g, n}(t)+\Gamma_{g, n} .
$$

Physically this reflects the fact that $\Delta_{g, n}$ has two kinds of boundaries. If we consider a fixed point $m$ in $\widetilde{\mathcal{K}}_{g, n}$ and move along the fiber direction, we encounter the boundary $\mathcal{C}_{g, n}(m)$, whose collection for all $m \in \widetilde{\mathcal{K}}$ gives the first term on the right hand side of (A.6). On the other hand if we move along $\widetilde{\mathcal{K}}_{g, n}$ then we may encounter the boundary $\partial \widetilde{\mathcal{K}}_{g, n}$. Thus the collection of $\mathcal{D}_{g, n}(m)$ for $m \in \partial \widetilde{\mathcal{K}}_{g, n}$ gives the second boundary of $\Delta_{g, n}$ represented by the $\Gamma_{g, n}$ term in (A.6).

As a consequence of the gluing compatibility relation (A.3) one can show that

$$
\Gamma_{g, n}=-\sum_{\substack{g_{1}, g_{1}, n_{1}, n_{2} \\ g_{1}+g_{2}=g, n_{1}+n_{2}=n+2}} \mathbf{S}\left[\left\{\mathcal{R}_{g_{1}, n_{1}}, \Delta_{g_{2}, n_{2}}\right\}\right] .
$$

Intuitively this means that $\Gamma_{g, n}$, which encodes the difference between $\mathcal{R}_{g, n}(t+\delta t)$ and $\mathcal{R}_{g, n}(t)$ at the boundary, gets contribution from two sources to order $\delta t$ - one that encodes the difference between $\mathcal{R}_{g_{1}, n_{1}}(t+\delta t)$ and $\mathcal{R}_{g_{1}, n_{1}}(t)$ and the other that encodes the difference between $\mathcal{R}_{g_{2}, n_{2}}(t+\delta t)$ and $\mathcal{R}_{g_{2}, n_{2}}(t)$. Since both give equal contributions after summing over $g_{1}, g_{2}, n_{1}, n_{2}$ we have kept only one of the terms and multiplied the result by a factor of 2 .

We are now ready to discuss the change in the action. This is given by

$$
\begin{aligned}
\delta S & =\delta t \sum_{g=0}^{\infty} g_{s}{ }^{2 g-2} \sum_{n=1}^{\infty} \frac{1}{n !} \int_{\delta \mathcal{R}_{g, n}(t)} \Omega_{6 g-6+2 n}^{(g, n)}\left(|\Psi\rangle^{\otimes n}\right) \\
& =\sum_{g=0}^{\infty} g_{s}{ }^{2 g-2} \sum_{n=1}^{\infty} \frac{1}{n !}\left[\int_{\Delta_{g, n}(t)} d \Omega_{6 g-6+2 n}^{(g, n)}\left(|\Psi\rangle^{\otimes n}\right)-\int_{\Gamma_{g, n}} \Omega_{6 g-6+2 n}^{(g, n)}\left(|\Psi\rangle^{\otimes n}\right)\right]
\end{aligned}
$$

where in arriving that the last expression we have used (A.6). This is the equation analogous to (3.28). Since this change is infinitesimal we can proceed as earlier and show that this change in the action can be reinterpreted as the result of a field redefinition of the form $|\Psi\rangle$ to $|\Psi\rangle+|\tilde{\delta} \Psi\rangle$ where $|\tilde{\delta} \Psi\rangle$ is given by

$$
\left\langle\Phi\left|c_{0}^{-}\right| \tilde{\delta} \Psi\right\rangle=-\sum_{g=0}^{\infty} g_{s}{ }^{2 g} \sum_{n=1}^{\infty} \frac{1}{(n-1) !} \int_{\Delta_{g, n}(t)} \Omega_{6 g-5+2 n}^{(g, n)}\left(|\Phi\rangle,|\Psi\rangle^{\otimes(n-1)}\right),
$$


for any state $|\Phi\rangle$ in $\mathcal{H}_{0}$. The proof of this follows in a straightforward fashion, with the change in the term proportional to $\left\langle\Psi\left|c_{0}^{-} Q_{B}\right| \Psi\right\rangle$ in the action matching the first term on the right hand side of (A.8) and the change in the rest of the terms in the action matching the second term on the right hand side of (A.8).

The important aspect of (A.9) is that even though the subspace $\Delta_{g, n}$ contains spurious singularities, the rules of vertical integration given in [18] give a procedure for integrating $\Omega_{6 g-5+2 n}^{(g, n)}$ over $\Delta_{g, n}$ yielding a finite result. Thus the field redefinition described in (A.9) is finite.

Open Access. This article is distributed under the terms of the Creative Commons Attribution License (CC-BY 4.0), which permits any use, distribution and reproduction in any medium, provided the original author(s) and source are credited.

\section{References}

[1] E. Witten, Interacting Field Theory of Open Superstrings, Nucl. Phys. B 276 (1986) 291 [INSPIRE].

[2] R. Saroja and A. Sen, Picture changing operators in closed fermionic string field theory, Phys. Lett. B 286 (1992) 256 [hep-th/9202087] [INSPIRE].

[3] N. Berkovits, SuperPoincaré invariant superstring field theory, Nucl. Phys. B 450 (1995) 90 [hep-th/9503099] [INSPIRE].

[4] N. Berkovits, The Ramond sector of open superstring field theory, JHEP 11 (2001) 047 [hep-th/0109100] [INSPIRE].

[5] Y. Okawa and B. Zwiebach, Heterotic string field theory, JHEP 07 (2004) 042 [hep-th/0406212] [INSPIRE].

[6] N. Berkovits, Y. Okawa and B. Zwiebach, WZW-like action for heterotic string field theory, JHEP 11 (2004) 038 [hep-th/0409018] [INSPIRE].

[7] M. Kroyter, Superstring field theory in the democratic picture, Adv. Theor. Math. Phys. 15 (2011) 741 [arXiv:0911.2962] [inSPIRE].

[8] M. Kroyter, Y. Okawa, M. Schnabl, S. Torii and B. Zwiebach, Open superstring field theory I: gauge fixing, ghost structure and propagator, JHEP 03 (2012) 030 [arXiv:1201.1761] [INSPIRE].

[9] B. Jurčo and K. Muenster, Type II Superstring Field Theory: Geometric Approach and Operadic Description, JHEP 04 (2013) 126 [arXiv:1303.2323] [INSPIRE].

[10] Y. Iimori, T. Noumi, Y. Okawa and S. Torii, From the Berkovits formulation to the Witten formulation in open superstring field theory, JHEP 03 (2014) 044 [arXiv:1312.1677] [INSPIRE].

[11] T. Erler, S. Konopka and I. Sachs, Resolving Witten's superstring field theory, JHEP 04 (2014) 150 [arXiv: 1312.2948] [INSPIRE].

[12] T. Erler, S. Konopka and I. Sachs, NS-NS Sector of Closed Superstring Field Theory, JHEP 08 (2014) 158 [arXiv: 1403.0940] [INSPIRE].

[13] H. Kunitomo, The Ramond Sector of Heterotic String Field Theory, PTEP 2014 (2014) 043B01 [arXiv:1312.7197] [INSPIRE]. 
[14] T. Erler, S. Konopka and I. Sachs, NS-NS Sector of Closed Superstring Field Theory, JHEP 08 (2014) 158 [arXiv: 1403.0940] [INSPIRE].

[15] R. Pius, A. Rudra and A. Sen, Mass Renormalization in String Theory: Special States, JHEP 07 (2014) 058 [arXiv: 1311.1257] [INSPIRE].

[16] R. Pius, A. Rudra and A. Sen, Mass Renormalization in String Theory: General States, JHEP 07 (2014) 062 [arXiv: 1401.7014] [INSPIRE].

[17] R. Pius, A. Rudra and A. Sen, String Perturbation Theory Around Dynamically Shifted Vacuum, JHEP 1410 (2014) 70 [arXiv:1404.6254] [INSPIRE].

[18] A. Sen, Off-shell Amplitudes in Superstring Theory, Fortsch. Phys. 63 (2015) 149 [arXiv: 1408.0571] [INSPIRE].

[19] D. Friedan, E.J. Martinec and S.H. Shenker, Conformal Invariance, Supersymmetry and String Theory, Nucl. Phys. B 271 (1986) 93 [INSPIRE].

[20] E.P. Verlinde and H.L. Verlinde, Multiloop Calculations in Covariant Superstring Theory, Phys. Lett. B 192 (1987) 95 [INSPIRE].

[21] O. Lechtenfeld, Superconformal ghost correlations on Riemann surfaces, Phys. Lett. B 232 (1989) 193 [inSPIRE].

[22] A. Morozov, Straightforward proof of Lechtenfeld's formula for beta, gamma correlator, Phys. Lett. B 234 (1990) 15 [INSPIRE].

[23] A. Belopolsky, De Rham cohomology of the supermanifolds and superstring BRST cohomology, Phys. Lett. B 403 (1997) 47 [hep-th/9609220] [INSPIRE].

[24] A. Belopolsky, New geometrical approach to superstrings, hep-th/9703183 [INSPIRE].

[25] A. Belopolsky, Picture changing operators in supergeometry and superstring theory, hep-th/9706033 [INSPIRE].

[26] E. D'Hoker and D.H. Phong, Two loop superstrings. 1. Main formulas, Phys. Lett. B 529 (2002) 241 [hep-th/0110247] [INSPIRE].

[27] E. D'Hoker and D.H. Phong, Two loop superstrings. 2. The Chiral measure on moduli space, Nucl. Phys. B 636 (2002) 3 [hep-th/0110283] [INSPIRE].

[28] E. D'Hoker and D.H. Phong, Two loop superstrings. 3. Slice independence and absence of ambiguities, Nucl. Phys. B 636 (2002) 61 [hep-th/0111016] [INSPIRE].

[29] E. D'Hoker and D.H. Phong, Two loop superstrings 4: The Cosmological constant and modular forms, Nucl. Phys. B 639 (2002) 129 [hep-th/0111040] [InSPIRE].

[30] E. D'Hoker and D.H. Phong, Two-loop superstrings. V. Gauge slice independence of the N-point function, Nucl. Phys. B 715 (2005) 91 [hep-th/0501196] [INSPIRE].

[31] E. D'Hoker and D.H. Phong, Two-loop superstrings VI: Non-renormalization theorems and the 4-point function, Nucl. Phys. B 715 (2005) 3 [hep-th/0501197] [INSPIRE].

[32] E. D'Hoker and D.H. Phong, Two-Loop Superstrings. VII. Cohomology of Chiral Amplitudes, Nucl. Phys. B 804 (2008) 421 [arXiv:0711.4314] [inSPIRE].

[33] E. Witten, Superstring Perturbation Theory Revisited, arXiv:1209.5461 [INSPIRE].

[34] E. Witten, More On Superstring Perturbation Theory, arXiv:1304.2832 [INSPIRE].

[35] E. Witten, Notes On Supermanifolds and Integration, arXiv:1209.2199 [INSPIRE]. 
[36] E. Witten, Notes On Super Riemann Surfaces And Their Moduli, arXiv:1209.2459 [INSPIRE].

[37] E. Witten, Notes On Holomorphic String And Superstring Theory Measures Of Low Genus, arXiv:1306.3621 [INSPIRE].

[38] R. Donagi and E. Witten, Supermoduli Space Is Not Projected, arXiv:1304.7798 [INSPIRE].

[39] R. Donagi and E. Witten, Super Atiyah classes and obstructions to splitting of supermoduli space, arXiv: 1404.6257 [INSPIRE].

[40] E. D'Hoker and D.H. Phong, Two-loop vacuum energy for Calabi-Yau orbifold models, Nucl. Phys. B 877 (2013) 343 [arXiv: 1307.1749] [INSPIRE].

[41] E. D'Hoker, Topics in Two-Loop Superstring Perturbation Theory, arXiv:1403.5494 [INSPIRE].

[42] J.J. Atick, G.W. Moore and A. Sen, Catoptric tadpoles, Nucl. Phys. B 307 (1988) 221 [INSPIRE].

[43] E. Witten, Noncommutative Geometry and String Field Theory, Nucl. Phys. B 268 (1986) 253 [inSPIRE].

[44] M. Saadi and B. Zwiebach, Closed String Field Theory from Polyhedra, Annals Phys. 192 (1989) 213 [INSPIRE].

[45] T. Kugo, H. Kunitomo and K. Suehiro, Nonpolynomial Closed String Field Theory, Phys. Lett. B 226 (1989) 48 [inSPIRE].

[46] T. Kugo and K. Suehiro, Nonpolynomial Closed String Field Theory: Action and Its Gauge Invariance, Nucl. Phys. B 337 (1990) 434 [InSPIRE].

[47] B. Zwiebach, Closed string field theory: Quantum action and the B-V master equation, Nucl. Phys. B 390 (1993) 33 [hep-th/9206084] [InSPIRE].

[48] A. Sen, Equations of Motion in Nonpolynomial Closed String Field Theory and Conformal Invariance of Two-dimensional Field Theories, Phys. Lett. B 241 (1990) 350 [INSPIRE].

[49] H. Hata and B. Zwiebach, Developing the covariant Batalin-Vilkovisky approach to string theory, Annals Phys. 229 (1994) 177 [hep-th/9301097] [INSPIRE].

[50] P.C. Nelson, Covariant Insertion of General Vertex Operators, Phys. Rev. Lett. 62 (1989) 993 [INSPIRE].

[51] A. Sen and E. Witten, unpublished.

[52] A. Berera, Unitary string amplitudes, Nucl. Phys. B 411 (1994) 157 [INSPIRE].

[53] E. Witten, The Feynman i€ in String Theory, JHEP 04 (2015) 055 [arXiv:1307.5124] [INSPIRE].

[54] S. Mukherji and A. Sen, Some all order classical solutions in nonpolynomial closed string field theory, Nucl. Phys. B 363 (1991) 639 [InSPIRE]. 